\title{
4D Atlas: Statistical Analysis of the Spatiotemporal Variability in Longitudinal 3D Shape Data
}

This paper was downloaded from TechRxiv (https://www.techrxiv.org).

\section{LICENSE}

CC BY 4.0

SUBMISSION DATE / POSTED DATE

$23-01-2021 / 31-01-2021$

\section{CITATION}

LAGA, HAMID; Padilla, Marcel; Jermyn, Ian H.; Kurtek, Sebastian; Bennamoun, Mohammed; Srivastava, Anuj (2021): 4D Atlas: Statistical Analysis of the Spatiotemporal Variability in Longitudinal 3D Shape Data. TechRxiv. Preprint. https://doi.org/10.36227/techrxiv.13629260.v1

$\mathrm{DOI}$ 


\title{
4D Atlas: Statistical Analysis of the Spatiotemporal Variability in Longitudinal 3D Shape Data
}

\author{
Hamid Laga, Marcel Padilla, Ian H. Jermyn, Sebastian Kurtek, Mohammed Bennamoun Senior \\ Member, IEEE Anuj Srivastava
}

\begin{abstract}
We propose a novel framework to learn the spatiotemporal variability in longitudinal 3D shape data sets, which contain observations of subjects that evolve and deform over time. This problem is challenging since surfaces come with arbitrary parameterizations and thus, they need to be spatially registered onto each others. Also, different deforming subjects, hereinafter referred to as 4D surfaces, evolve at different speeds and thus, they need to be temporally aligned onto each others. We solve this spatiotemporal registration problem using a Riemannian approach. We treat a 3D surface as a point in a shape space equipped with an elastic Riemmanian metric that measures the amount of bending and stretching that the surfaces undergo. A 4D surface can then be seen as a trajectory in this space. With this formulation, the statistical analysis of 4D surfaces can be casted as the problem of analyzing trajectories, or 1D curves, embedded in a nonlinear Riemannian manifold. However, performing the spatiotemporal registration, and subsequently computing statistics, on such nonlinear spaces is not straightforward as they rely on complex nonlinear optimizations. Our core contribution is the mapping of the surfaces to the space of Square-Root Normal Fields (SRNF) where the $\mathbb{L}^{2}$ metric is equivalent to the partial elastic metric in the space of surfaces. Thus, by solving the spatial registration in the SRNF space, the problem of analyzing 4D surfaces becomes the problem of analyzing trajectories embedded in the SRNF space, which has a Euclidean structure. In this paper, we develop the building blocks that enable such analysis. These include: (1) the spatiotemporal registration of arbitrarily parameterized 4D surfaces even in the presence of large elastic deformations and large variations in their execution rates, (2) the computation of geodesics between 4D surfaces, (3) the computation of statistical summaries, such as means and modes of variation, of collections of $4 \mathrm{D}$ surfaces, and (4) the synthesis of random $4 \mathrm{D}$ surfaces. We demonstrate the utility and performance of the proposed framework using 4D facial surfaces and 4D human body shapes.
\end{abstract}

Index Terms-Dynamic surfaces, Elastic metrics, Square-Root Normal Field, Statistical summaries, Shape synthesis and generation, 4D surface, Human4D, Face4D.

\section{INTRODUCTION}

HAPE, an important property of natural and man-made S3 objects, deforms over time as a result of many internal and external factors. For instance, anatomical organs such as bones, kidneys, and subcortical structures in the brain deform as a result of growth and/or disease progression; human faces deform as a consequence of talking, executing facial expressions, and aging. Similarly, actions and motions such as walking, jumping, and running are the result of deformations, over time, of the human body

- Hamid Laga is with the Information Technology Discipline, Murdoch University, Murdoch, 6150 (Australia), with the Harry Butler Institute, Murdoch University, Murdoch, 6150 (Australia), Email: H.Laga@murdoch.edu.au, and with the University of South Australia, The Phenomics and Bioinformatics Research Centre, SA 5000, Australia.

- Marcel Padilla is with TU Berlin, Germany. Email: padilla@math.tuberlin.de

- Ian H. Jermyn is with Durham University. Email: i.h.jermyn@durham.ac.uk

- Sebastian Kurtek is with the Ohio State University, US. Email: kurtek.1@stat.osu.edu

- Mohammed Bennamoun is with the University of Western Australia, Perth, WA 6009, Australia. Email:mohammed.bennamoun@uwa.edu.au

- Anuj Srivasta is with Florida State University, US. Email: anuj@stat.fsu.edu

Manuscript received XXXX, 2020; revised XXX, 2020. shape. The ability to understand and model (1) the typical deformation patterns of a class of 3D objects and (2) the variability of these deformations within and across object classes has many applications in fields such as medical diagnosis and biological growth modelling, e.g., measuring the intensity of pain from facial deformations [1], and distinguishing between normal growth and disease progression from the deformation overtime of the body shape. In computer vision and graphics, the ability to statistically model such spatiotemporal variability can be used to summarize collections of 3D animations, and to synthesize and simulate animations and motions. Similar to 3D morphable face models [2], it can also be used as a generative model for synthesizing large corpora of labelled longitudinal 3D shape data, e.g., 4D faces, for training deep neural networks.

In this article, we propose a novel framework for the statistical analysis of longitudinal 3D shape data composed of subjects that deform over time. In what follows, we refer to a subject captured at different points in time, e.g., a 3D human face performing a facial expression or speaking a sentence, or a 3D human body shape growing or performing actions, as a $4 D$ (or $3 D+t$ surface). Given a collection of $4 \mathrm{D}$ surfaces, our goal is to:

- Compute the typical deformation pattern, i.e., the sta- 
tistical mean 4D surface. For example, the same person can smile in different ways. Similarly, different people smile differently. The goal is to learn, based on observed longitudinal shape data, the typical smile.

- Compute the main modes of variation, analogous to Principal Component Analysis (PCA), but for modelling variability in 4D surface collections, unlike previous work [3], [4], which only focused on 3D shape variability.

- Characterize a population of 4D surfaces using statistical models.

- Synthesize new 4D surfaces by sampling, randomly or in a controlled fashion, from these statistical models.

We refer to these tasks as the process of constructing a $4 \mathrm{D}$ atlas. Achieving this goal requires solving important fundamental challenges. In fact, 3D objects such as faces, human body shapes, and anatomical organs, which come with arbitrary parameterizations, exhibit large elastic deformations within the same subject and across different subjects. This makes their spatial registration, i.e., finding one-to-one correspondences between each pair of shapes very challenging. In the case of $4 \mathrm{D}$ surfaces, there is an additional temporal variability due to different execution rates (speeds) of evolution within and across subjects. For instance, a walking action can be executed at variable speeds by even the same person. Thus, the statistical analysis of the spatiotemporal variability in samples of 4D surfaces requires efficient spatiotemporal registration of these samples. Spatial registration refers to the process of finding a one-toone correspondence between two 3D surfaces of the same object, captured at different points in time, or of different individuals. Temporal registration refers to the problem of finding the optimal time warping that aligns 4D surfaces, e.g., walking actions, performed at different execution rates.

We treat a $4 \mathrm{D}$ surface as a trajectory in a high dimensional nonlinear space. We then formulate the problem of anlayzing the spatiotemporal variability of $4 \mathrm{D}$ surfaces as the problem of the statistical analysis of elastic trajectories, where elasticity corresponds to variations in the execution rates of the $4 \mathrm{D}$ surfaces. However, performing statistics on trajectories embedded in nonlinear spaces of high dimension is computationally expensive since it relies on nonlinear optimizations. Our core contribution in this article is the mapping of the surfaces to the space of Square-Root Normal Fields (SRNF) [4], [5], which has a Euclidean structure, see Section 3.1 In particular, the $\mathbb{L}^{2}$ metric in the space of SRNFs is equivalent to the partial elastic metric in the space of surfaces. As such, the problem of analyzing 4D surfaces becomes the problem of analyzing trajectories, or curves, embedded in the Euclidean space of SRNFs.

This article develops the building blocks that enable such analysis. These include the spatiotemporal registration of $4 \mathrm{D}$ surfaces, even in the presence of large elastic deformations and large variations in their execution rates, and the computation of geodesics between 4D surfaces. We use these building blocks for the computation of statistical summaries, such as means and modes of variation of collections of $4 \mathrm{D}$ surfaces, and for the synthesis, either randomly or in a controlled fashion, of 4D surfaces. We demonstrate the utility and performance of the proposed framework using 4D facial surfaces from the VOCA dataset [6], 4D human body shapes from the Dynamic FAUST (DFAUST) dataset [7], and dressed 4D human body shapes from the CAPE dataset [8]. Our approach is, however, general and can be applied to any type of spherically-parameterized surfaces. In summary, the main contributions of this article are;

- We represent 4D surfaces as trajectories in the space of SRNFs, which has a Euclidean structure (Section 3.1). This key contribution enables the usage of standard computational tools for the analysis and modelling of 4D surfaces (Section 3.2.

- We propose efficient algorithms for the spatiotemporal registration of $4 \mathrm{D}$ surfaces and for the computation of geodesics between such 4D surfaces, even in the presence of large elastic deformations and large variations in execution rates (Sections 3.2.2 and 3.2.3.

- The framework does not assume, neither explicitly nor implicitly, that the correspondences between the surfaces are given. It solves simultaneously for the spatial and temporal registrations, and for the $4 \mathrm{D}$ geodesics that are optimal under the proposed metrics.

- We develop computational tools for computing summary statistics of $4 \mathrm{D}$ surfaces, and for synthesizing $4 \mathrm{D}$ surfaces from formal statistical models (Section 4).

The remainder of this paper is organized as follows. We first discuss the related work in Section 2 Section 3 describes the proposed mathematical framework. Section 4 discusses its application to various statistical analysis tasks. Section 5 presents the results and discusses the performance of the proposed framework. Section 6 summarizes the main findings of this paper and discusses future research directions.

\section{Related Work}

We classify the state-of-the-art into two categories. Methods in the first category focus on cross-sectional shape data (Section 2.1). Methods in the second category focus on longitudinal shape data by taking into account the temporal dimension (Section 2.2).

\subsection{Statistical models of cross-section 3D shape data}

Modelling and exploring shape variability in collections of 2D and 3D objects have been extensively studied in the literature. Early methods use Principal Component Analysis (PCA) to characterize the shape space of objects. Initially introduced for the analysis of planar shapes, the active shape models of Cootes et al. |9| have been extended to the analysis of 3D faces [10] and 3D human bodies [3], see also |2] for a detailed survey. These methods represent 3D objects as discrete sets of landmarks, e.g., vertices, which are assumed to be in correspondence across a population of objects, and use standard Euclidean metrics for their comparison. As a result, these approaches are limited to 3D objects that undergo small elastic deformations.

To handle large nonlinear variations, e.g., elastic deformations such as the bending and stretching observed in 3D human body shapes, Anguelov et al. |11| introduced SCAPE, which represents body shape and pose-dependent shape in terms of triangle deformations instead of vertex displacements. Hasler et al. [12] learn two linear models: one for pose and one for body shape. Loper et al. [13] introduce 
SMPL, a vertex-based linear model for human body shape and pose-dependent shape variation. This model, which has been extensively used in the literature for the analysis of the human body shape, has been extended to various types of objects such as animals [14] and human body parts [15]. While these models are able to capture large variations, they exhibit two fundamental limitations. First, they rely on separate models for pose-independent shape, pose-dependent shape, and pose. As a result, they are limited to specific classes of objects, e.g., human bodies. Changing the target application, e.g., to animals [14] or infants [16], requires redefining the model. Second, and more importantly, they either assume a given registration between the surfaces of the $3 \mathrm{D}$ objects or solve the registration problem separately by matching vertices across the surfaces using an unrelated optimization criterion.

Recently, there has been a growing interest in analyzing variability in 3D shape collections using tools from differential and Riemannian geometry [4], [5], [17], [18], |19], [20], [21], see also [22] for a detailed survey. The most relevant work to this article is the Square-Root Normal Field (SRNF) representation introduced by Jermyn et al. [5]. In this work, differences between parameterized surfaces are measured using a partial elastic Riemannian metric defined as a weighted sum of a bending term and a stretching term. More importantly, Jermyn et al. [5] show that by carefully choosing the weights of these two terms, the complex partial elastic metric reduces to the $\mathbb{L}^{2}$ metric in the space of SRNFs. Thus, by treating shapes of objects as points in the SRNF space, a straight line between two points in this space is equivalent to the geodesic (or shortest) curve in the original space of surfaces under the partial elastic metric, and represents the optimal deformation between them. As a result, one can perform the statistical analysis tasks in the SRNF space using standard vector calculus, and then map the results back to the space of surfaces (for visualization), using the numerical inversion method of Laga et al. [4]. Another important property of the SRNF representation is that both registration and optimal deformation (geodesic) can be computed jointly, using the same partial elastic metric.

Note that one of the fundamental problems in statistical shape analysis is correspondence and registration. These have been extensively investigated, see for example [23] for a detailed survey. These methods, however, do not define a shape space and a metric that enable the computation of geodesics and statistics. Also, correspondence methods that are based on the intrinsic properties of surfaces, e.g., Generalized Multidimensional Scaling [24], spectral descriptors [25], or functional maps, which rely on given input descriptors [26], [27], are suitable for surfaces that deform in an isometric manner, i.e., only bend but do not stretch. They also require manual input, e.g., landmarks, to solve the symmetry ambiguity.

\subsection{Statistical models for longitudinal shape data}

Early works focused on longitudinal 2D shape data. For instance, Anirudh et al. [28] represent the contour of planar shapes that evolve over time as trajectories on a Grassmann manifold, and use the Transported Square-Root Velocity Function (TSRVF) representation for their rate-invariant analysis. This approach has been later extended to the analysis of the trajectories of sparse features or landmarks measured on the surface of a deforming 3D subject. For instance, Anirudh et al. [28], and later Ben Amor et al. [29], represent human body actions using dynamic skeletons. By treating each skeleton, represented by a set of landmarks, as a high-dimensional point on Kendall's shape space [30], motions become trajectories in a high-dimensional, Euclidean space. Thus, one can use the rich literature on statistical analysis of high-dimensional curves [31] to compute (1) rate-invariant comparisons of motion, (2) geodesics between actions, (3) summary statistics such as the average action or the principal modes of variability in a sample of actions, and (4) random (unobserved) sampling of skeletal motions from formal statistical models. Since a skeleton is represented as a set of landmarks, this approach can be extended to the analysis of the motion of landmarks on arbitrary surfaces, including human faces or anatomical organs. This approach, however, has two fundamental limitations. First, a skeleton is represented as a point on Kendall's shape space equipped with the $\mathbb{L}^{2}$ metric, which is not suitable for large articulated motions. Also, it does not capture surface elasticity, and thus, cannot be used to model growth processes and surface deformations due to motion. Second, the use of landmarks to represent the objects of interest makes it unsuitable for modelling the dynamics of an entire surface. While this can be addressed by using two separate models, one for shape and another for motion, such an approach will fail to capture motion-dependent shape variations.

Debavelaere et al. [32] and Bone et al. [33] introduced an approach for the statistical analysis of longitudinal 2D and $3 \mathrm{D}$ shape data that is based on the use the Large Deformation Diffeomorphic Metric Mapping [34]. The idea is to represent a $4 \mathrm{D}$ surface as a flow of deformations of the $3 \mathrm{D}$ volume around each surface and then code deformations as geodesics on a Riemannian manifold. However, in general, natural deformations do not correspond to geodesics but can be arbitrary paths on the shape space. Also, deforming $3 \mathrm{D}$ volumes is expensive in terms of computation and memory requirements. Finally, the approach relies on manually specified landmarks to efficiently register the 3D volumes. The approach we propose in this paper works directly on surfaces, does not assume that deformations are (piecewise) geodesics, and does not rely on landmarks for the spatiotemporal registration. To handle large articulated and elastic motions, we map the surfaces of $3 \mathrm{D}$ objects to the space of SRNFs [5], which has a Euclidean structure, i.e., the complex partial elastic metric in the original space of surfaces is equivalent to the $\mathbb{L}^{2}$ metric in the SRNF space. With this representation, $4 \mathrm{D}$ surface analysis becomes the problem of analyzing trajectories, i.e., curves embedded in a high-dimensional space equipped with the $\mathbb{L}^{2}$ metric. In this paper, we develop all the required computational tools for the statistical analysis of such 4D surfaces, and demonstrate their efficiency on complex longitudinal 3D shape data such as $4 \mathrm{D}$ faces and $4 \mathrm{D}$ human body shapes.

\section{MATHEMATICAL FRAMEWORK}

We describe in this section the mathematical framework for the spatiotemporal registration and comparison of $4 \mathrm{D}$ 


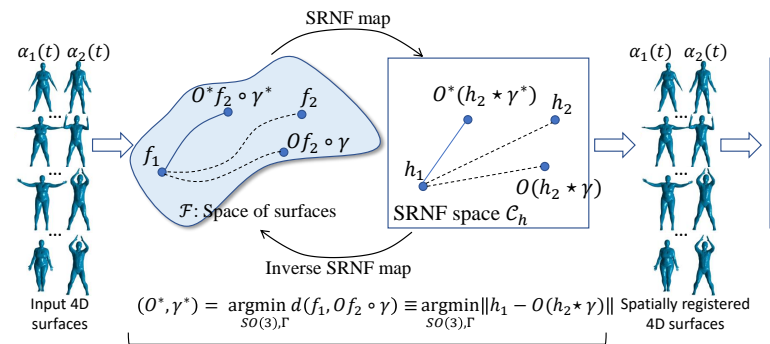

(a) Spatial elastic registration of surfaces

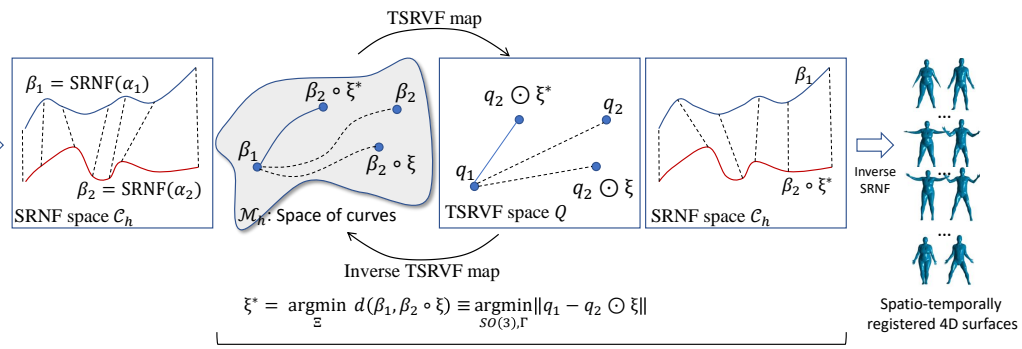

(b) Temporal registration of sequences

Fig. 1. Overview of the proposed framework. Surfaces are first mapped onto the space of Square Root Normal Fields (SRNF) and spatially registered using the $\mathbb{L}^{2}$ metric, which is equivalent to the partial elastic metric in the original space of surfaces. In the SRNF space, 4D surfaces are treated as curves, and then mapped into the space of Transported Square-Root Vector Fields (TSRVF) for their temporal registration. Points in the TSRVF space are mapped back to the space of SRNFs and then to the original space of surfaces for visualization.

surfaces (see Fig. 1 for an overview), and then discuss in Section 4 its application to various statistical analysis tasks. A $4 \mathrm{D}$ surface, where the fourth dimension refers to time, is a 3D surface that evolves over time. Examples of such 4D surfaces include facial expressions (e.g., a smiling face), a human body shape performing an action such as walking or jumping, or an anatomical organ which evolves over time due to natural growth or disease progression. It can be represented as a path $\alpha(t), t \in[0,1]$ such that $\alpha(0)$ and $\alpha(1)$ are the initial and final surfaces, respectively, and $\alpha(t), 0<t<1$ are the intermediate surfaces. The main challenges posed by the statistical analysis of such $4 \mathrm{D}$ surfaces are two-fold. First, surfaces within the same 4D surface and across different $4 \mathrm{D}$ surfaces come with arbitrary poses and registrations. Second, 4D surfaces can have different execution rates, e.g., two smiling expressions performed at different speeds. Thus, in order to compare and perform statistical analysis on samples of $4 \mathrm{D}$ surfaces, we first need to spatiotemporally register them onto each others.

We solve the spatiotemporal registration problem using tools from differential geometry. We treat surfaces as points in a Riemannian shape space equipped with an elastic metric that captures shape differences in terms of bending and stretching energies. We then formulate the elastic registration problem, i.e., the problem of computing spatial correspondences, as that of finding the optimal rotation and reparameterization that align one surface onto another. This enables comparing and spatially registering surfaces, even in the presence of large elastic deformations (Section 3.1).

With this representation, a $4 \mathrm{D}$ surface, i.e., a temporal sequence of $3 \mathrm{D}$ surfaces, becomes a time-parameterized trajectory in the above-referenced Riemannian shape space. Thus, the problem of statistical analysis of $4 \mathrm{D}$ surfaces is reduced to the problem of analyzing curves. Similar to surfaces, we define a space of curves and a Riemannian metric that allows temporal registration, also referred to as time warping, across two 4D surfaces. The chosen metric quantifies the amount of elastic deformations needed to temporally align $4 \mathrm{D}$ surfaces onto each others (Section 3.2.

\subsection{The elastic shape space of surfaces}

We consider a surface as a function $f$ of the form:

$$
f \quad: \quad \Omega \rightarrow \mathbb{R}^{3} ; s \mapsto f(s)=(X(s), Y(s), Z(s)),
$$

where $\Omega$ is a parameterization domain and $s \in \Omega$ is the parameter in this domain. The choice of the parameterization domain depends on the nature of the surfaces of interest. When dealing with closed surfaces of genus- $0, \Omega$ can be a unit sphere, i.e., $\Omega=\mathbb{S}^{2}$, and $s=(u, v), u \in[0, \pi]$ and $v \in[0,2 \pi[$, is the spherical coordinates, defined on the unit sphere. When dealing with open surfaces that are homeomorphic to a disk, e.g., human faces, then $\Omega$ can be chosen to be $[0,1] \times[0,2 \pi]$.

To remove shape-preserving transformations, we first normalize the surfaces for translation and scale by translating them so that their center of mass is located at the origin, and then scaling them to have unit surface area. The space of the normalized surfaces, hereinafter denoted by $\mathcal{F}$, is called the preshape space.

Having removed translation and scale, we still need to account for rotations and reparameterizations. Those are handled algebraically. For any surface $f \in \mathcal{F}$ and for any rotation $O \in S O(3), O f$ and $f$ have equivalent shapes. Similarly, any reparameterization of a surface with an orientation-preserving diffeomorphism preserves its shape. Let $\Gamma$ be the space of all orientation-preserving diffeomorphisms of $\Omega$. Then, $\forall \gamma \in \Gamma, f$ and $f \circ \gamma$, i.e., the reparameterization of $f$ with $\gamma$, have the same shape. Note also that reparameterizations provide dense correspondences across surfaces.(Here, $\circ$ refers to the composition of two functions.) That is, if one wants to put a surface $f_{2}$ in correspondence with another surface $f_{1}$, then one only needs to find a rotation $O^{*}$ and a reparameterization $\gamma^{*}$ such that $O^{*}\left(f_{2} \circ \gamma^{*}\right)$ is as close as possible to $f_{1}$. This is precisely the process of $3 \mathrm{D}$ surface registration, and is defined mathematically as:

$$
\left(O^{*}, \gamma^{*}\right)=\underset{O \in S O(3), \gamma \in \Gamma}{\operatorname{argmin}} d_{\mathcal{F}}\left(f_{1}, O\left(f_{2} \circ \gamma\right)\right),
$$

where $d_{\mathcal{F}}$ is a measure of distances between surfaces in $\mathcal{F}$.

\subsubsection{SRNF representation of surfaces}

For efficient registration and comparison of surfaces, the distance measure, or metric, $d_{\mathcal{F}}$ should quantify interpretable shape differences, i.e., the amount of bending and stretching one needs to apply on one surface to deform it into another. It should also be simple enough to facilitate the efficient computation of correspondences and geodesic paths. Jermyn et al. [5] introduced a partial elastic metric that measures differences between surfaces as a weighted 


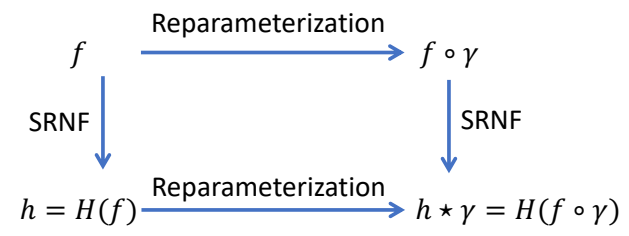

Fig. 2. SRNF map and reparameterization of surfaces.

sum of the amount of bending and stretching that one needs to apply to a surface in order to align it onto another. In this approach, bending is measured in terms of changes in the orientation of the unit normal vectors, while stretching is measured in terms of changes in the infinitesimal surface areas. More importantly, Jermyn et al. [5] showed that by using a special representation of surfaces, called the SquareRoot Normal Field (SRNF), the complex partial elastic metric reduces to the simple $\mathbb{L}^{2}$ metric on the SRNF space.

Definition 3.1 (SRNF maps). The SRNF map $H(f)$ of a surface $f \in \mathcal{F}$ is defined as the normal vector field of the surface scaled by the square-root of the local area around each surface point:

$$
\begin{aligned}
H: \mathcal{F} & \rightarrow \mathcal{C}_{h} \\
f & \mapsto H(f)=h, \text { such that } h(u, v)=\frac{\mathbf{n}(u, v)}{\|\mathbf{n}(u, v)\|_{2}^{\frac{1}{2}}},
\end{aligned}
$$

where $\mathcal{C}_{h}$ is the space of all SRNFs, $\mathbf{n}=\frac{\partial f}{\partial u} \times \frac{\partial f}{\partial v}$ is the normal field to the surface $f$ and $\|\cdot\|_{2}$ is the Euclidean norm in $\mathbb{R}^{3}$.

The SNRF representation of surfaces has nice properties that make it suitable for the various analysis tasks at hand:

- The SRNF is translation and scale invariant. Also, the SRNF of a rotated surface is simply the rotation of the SRNF of that surface, i.e., $H(O f)=O H(f)$.

- $\forall \gamma \in \Gamma, H(f \circ \gamma)=\sqrt{\left|J_{\gamma}\right|}(h \circ \gamma) \equiv h * \gamma$, where $J_{\gamma}$ is the Jacobian of $\gamma$ and $|\cdot|$ is its determinant; see Fig. 2 .

- Under the $\mathbb{L}^{2}$ metric in the space of SRNFs, the action of $\Gamma$ is by isometries, i.e., $\forall \gamma \in \Gamma$ and $\forall f_{1}, f_{2} \in \mathcal{F}, \| h_{1}-$ $h_{2}\|=\| h_{1} * \gamma-h_{2} * \gamma \|$, where $h_{i}=H\left(f_{i}\right), i=1,2$.

- The space of SRNFs is a subset of $\mathbb{L}^{2}\left(\Omega, \mathbb{R}^{3}\right)$. In addition, the $\mathbb{L}^{2}$ metric in $\mathcal{C}_{h}$ is equivalent to the partial elastic metric in the space of surfaces. As such, geodesic curves in the space of surfaces $\mathcal{F}$ become straight lines in the SRNF space $\mathcal{C}_{h}$, see Fig. 1. (a).

- While there is no analytical expression for the inverse SRNF map, and in fact, the injectivity and surjectivity of the SRNF remain open questions, Laga et al. [4] showed that, for a given SRNF map of a valid surface, one can always retrieve the original surface, up to a translation, using an efficient numerical inversion procedure [4].

The last three properties are critical for comparison and atlas construction of $4 \mathrm{D}$ surfaces. In fact, one can perform elastic registration of surfaces using the standard $\mathbb{L}^{2}$ metric in the space of SRNFs, which is computationally very efficient compared to using the complex elastic metric in the space of surfaces (Section 3.1.2). Further, temporal evolutions of surfaces (e.g., a facial expression or an aging anatomical organ) can be interpreted as curves in the Euclidean space of SRNFs, making them amenable to statistical analysis. Thus, the problem of constructing $4 \mathrm{D}$ atlases can be reduced to the problem of statistical analysis of elastic curves in the space of SRNFs using standard statistical tools developed for Euclidean spaces. After analysis, the results can be mapped back to the original space of surfaces using efficient SRNF inversion procedures [4] (Section 3.2.

\subsubsection{Spatial elastic registration of surfaces}

Under the SRNF representation, the elastic registration problem in Eqn. (2) can be reformulated using the $\mathbb{L}^{2}$ metric on $\mathcal{C}_{h}$, the space of SRNFs, instead of the complex partial elastic metric on the preshape space $\mathcal{F}$. Let $f_{1}$ and $f_{2}$ be two surfaces in the preshape space $\mathcal{F}$, and $h_{1}$ and $h_{2}$ their SRNFs. Then, the rotation and reparameterization that optimally register $f_{2}$ to $f_{1}$ are given by:

$$
\left(O^{*}, \gamma^{*}\right)=\underset{O \in S O(3), \gamma \in \Gamma}{\operatorname{argmin}}\left\|h_{1}-O\left(h_{2} * \gamma\right)\right\|,
$$

where $*$ is the composition operator between an SRNF and a diffeomorphism $\gamma \in \Gamma$. This joint optimization over $S O(3)$ and $\Gamma$ can be solved by alternating between the two marginal optimizations (this is allowed due to the product structure of $S O(3) \times \Gamma)$ |35|:

- Assuming a fixed parameterization, solve for the optimal rotation using Procrustes analysis via Singular Value Decomposition (SVD).

- Assuming a fixed rotation, solve for the optimal reparameterization using a gradient descent algorithm.

These two steps are repeated a few times until convergence. Although this registration approach converges to a local optima, in practice, it can be used in a very efficient way. In fact, since a $4 \mathrm{D}$ surface $\alpha$ is given as a sequence of discrete realizations $\alpha\left(t_{i}\right) \in \mathcal{F}, i=0, \cdots, n$, with $t_{0}=0$ and $t_{n}=1$, one can perform the elastic registration sequentially. Let $\beta=H(\alpha)$ be the SRNF map of the $4 \mathrm{D}$ surface $\alpha$, i.e., $\forall t \in[0,1], \beta(t)=H(\alpha(t))$. Also let $\alpha_{0}$ be a reference surface randomly chosen from the population of surfaces being analyzed, and $\beta_{0}$ its SRNF map ( $\alpha_{0}$ can be, for example, $\alpha(0))$. Then,

1) Find $O_{0} \in S O(3)$ and $\gamma_{0} \in \Gamma$ that register $\beta\left(t_{0}=0\right)$ (the start point of SRNF path) to the SRNF of the reference surface $\beta_{0}$, by solving Eqn. (4).

2) For $i=0, \ldots, n$, - $\beta\left(t_{i}\right) \leftarrow O_{0}\left(\beta\left(t_{i}\right) * \gamma_{0}\right)$ and $\alpha\left(t_{i}\right) \leftarrow O_{0} \alpha\left(t_{i}\right) \circ \gamma_{0}$.

3) For $i=1, \ldots, n$,

- Find, by solving Eqn. (4), $O_{i} \in S O(3)$ and $\gamma_{i} \in \Gamma$ that register $\beta\left(t_{i}\right)$ to $\beta\left(t_{i-1}\right)$.

- $\beta\left(t_{i}\right) \leftarrow O_{i}\left(\beta\left(t_{i}\right) * \gamma_{i}\right)$ and $\alpha\left(t_{i}\right) \leftarrow O_{i} \alpha\left(t_{i}\right) \circ \gamma_{i}$.

The first step ensures that, when we are given a collection of $4 \mathrm{D}$ surfaces $\alpha_{j}, j=1, \cdots, n$, the surfaces $\alpha_{j}(0), j=$ $1, \cdots, n$ are registered onto each others. The subsequent steps ensure that $\forall t, \alpha_{j}(t)$ is registered onto $\alpha_{j}(0)$. This sequential approach is efficient since, in general, elastic deformations between two consecutive frames in a $4 \mathrm{D}$ surface are relatively small. In what follows, we assume that all surfaces within a $4 \mathrm{D}$ surface and across $4 \mathrm{D}$ surfaces are correctly registered, i.e., they have been normalized for translation and scale, and optimally rotated and reparameterized using the approach described in this section. 


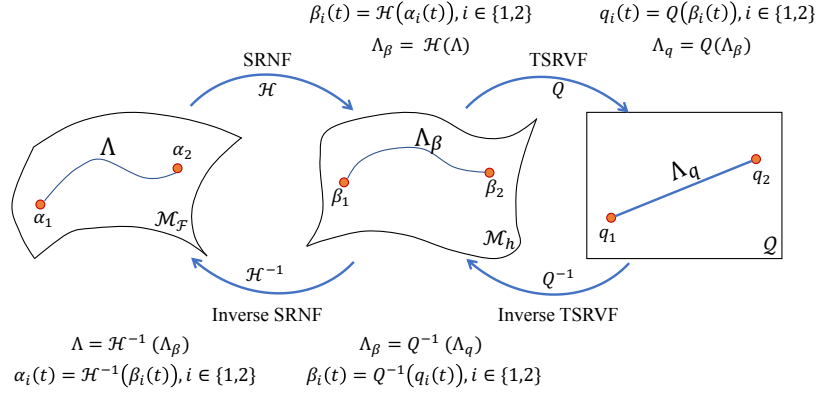

Fig. 3. To statistically analyze 4D surfaces $\alpha_{i}$, we first compute their SRNF maps $\beta_{i}=H\left(\alpha_{i}\right)$, and then map them to the TSRVF space, i.e., $q_{i}=Q\left(\beta_{i}\right)$. With this mapping, geodesics between 4D surfaces become straight lines in the space of TSRVFs $\mathcal{Q}$.

\subsection{The shape space of 4D surfaces}

Under the setup outlined in Section 3.1. a $4 \mathrm{D}$ surface is a curve $\alpha:[0,1] \rightarrow \mathcal{F}$. However, since $\mathcal{F}$ is endowed with the partial elastic metric is non-Euclidean, we propose to further map the $4 \mathrm{D}$ surfaces to the SRNF space, which has a Euclidean structure, and thus become curves of the form $\beta$ : $[0,1] \rightarrow \mathcal{C}_{h}$. With this representation, all statistical tasks are carried out in $\mathcal{C}_{h}$ under the $\mathbb{L}^{2}$ metric with results mapped back to the space of surfaces $\mathcal{F}$ afor visualization.

\subsubsection{TSRVF representation of SRNF trajectories}

Let $\alpha$ be a curve (path) in $\mathcal{F}$ and $\beta$ its image under the SRNF map, i.e., $\forall t \in[0,1], \beta(t)=H(\alpha(t))$; $\beta$ is also a curve, but in $\mathcal{C}_{h}$. Let $\mathcal{M}_{\mathcal{F}}$ be the space of all paths in $\mathcal{F}$, and $\mathcal{M}_{h}$ be the space of all paths in $\mathcal{C}_{h}: \mathcal{M}_{h}=\left\{\beta:[0,1] \rightarrow \mathcal{C}_{h} \mid \beta=\right.$ $H(\alpha), \alpha \in \mathcal{M}_{\mathcal{F}}$, see Fig. 3 .

To temporally register, compare, and summarize samples of such curves, we need to define an appropriate metric on $\mathcal{M}_{\mathcal{F}}$, or $\mathcal{M}_{h}$, that is invariant to the rate (or speed) of the $4 \mathrm{D}$ surfaces. For example, facial expressions that only differ in the rate of their execution should be deemed equivalent under such a metric. Let $\Xi=\{\xi:[0,1] \rightarrow[0,1] \mid 0<\dot{\xi}<\infty\}$ denote all reparameterizations of the temporal domain $[0,1]$. Here, $\dot{\xi}=\frac{d \xi}{d t}$. Then, for any $\xi \in \Xi, \beta \circ \xi$ and $\beta$ only differ in the rate of execution and are thus equivalent. The function $\xi$ is often referred to as a time warping of the domain $[0,1]$. Temporal registration of two $4 \mathrm{D}$ surfaces $\alpha_{1}$ and $\alpha_{2}$ then becomes the problem of registering their corresponding curves $\beta_{1}$ and $\beta_{2}$ in the SRNF space. This requires solving for an optimal reparameterization $\xi^{*} \in \Xi$ that minimizes an appropriate distance $d(\cdot, \cdot)$ between $\beta_{1}$ and $\beta_{2}$ :

$$
\xi^{*}=\underset{\xi \in \Xi}{\operatorname{argmin}} d\left(\beta_{1}, \beta_{2} \circ \xi\right) .
$$

The optimization over $\Xi$ in Eqn. (5) ensures rate invariance. Thus, we are left with defining a distance $d(\cdot, \cdot)$ that is invariant to time warping of the temporal domain $[0,1]$. To this end, we borrow tools from Srivastava et al. [31] for analyzing shapes of curves in $\mathbb{R}^{n}, n \geq 2$. The associated elastic metric defined therein is invariant to reparameterizations of curves, and quantifies the amount of bending and stretching of the curves in terms of changes in the orientations and lengths of their tangent vectors, respectively. However, instead of directly working with such a complex elastic metric, Su et al. [36] introduced the Transported Square-Root Vector
Field (TSRVF) representation, which simplifies the complex elastic metric for analyzing curves on $\mathcal{M}_{h}$ into the simple $\mathbb{L}^{2}$ metric, see Fig. 3

Definition 3.2 (Transported Square-Root Vector Field (TSRVF)). For any smooth trajectory $\beta \in \mathcal{M}_{h}$, the transported square-root vector field (TSRVF) is a parallel transport of a scaled velocity vector field of $\beta$ to a reference point $c \in \mathcal{C}_{h}$ according to

$$
Q(\beta)(t)=q(t)=\frac{\left.\dot{\beta}(t)\right|_{\beta(t) \rightarrow c}}{\sqrt{\|\dot{\beta}(t)\|}},
$$

where $\dot{\beta}=\frac{\partial \beta}{\partial t}$ is the tangent vector field on $\beta$ and $\|\cdot\|$ is the $\mathbb{L}^{2}$ metric on $\mathcal{C}_{h}$.

Note that the parallel transport $\left.\dot{\beta}(t)\right|_{\beta(t) \rightarrow c}$ is performed along the geodesic from $\beta(t)$ to $c$. The TSRVF representation has nice properties that facilitate efficient temporal registration of $4 \mathrm{D}$ surfaces. Let $\beta_{1}$ and $\beta_{2}$ be two trajectories on $\mathcal{M}_{h}$, and let $q_{1}$ and $q_{2}$ be their respective TSRVFs. Then,

- The elastic metric on the space of trajectories $\mathcal{M}_{h}$ reduces to the $\mathbb{L}^{2}$ metric on the space of their TSRVFs. Thus, one can use the $\mathbb{L}^{2}$ metric to compare two paths:

$$
d\left(\beta_{1}, \beta_{2}\right)=\left\|q_{1}-q_{2}\right\|=\left(\int_{0}^{1}\left\|q_{1}(t)-q_{2}(t)\right\|^{2} d t\right)^{\frac{1}{2}},
$$

where $\|\cdot\|$ is again the $\mathbb{L}^{2}$ norm on $\mathcal{C}_{h}$.

- For any $\xi \in \Xi, Q(\beta \circ \xi)=(q \circ \xi) \sqrt{\dot{\xi}(t)} \equiv q \odot \xi$. Here, $\odot$ is the composition operator between an SRVF $q$ and a diffeomorphism $\xi$.

- Under the $\mathbb{L}^{2}$ metric, the action of the reparameterization group $\Xi$ on the space of TSRVFs is by isometries, i.e., $\left\|q_{1}-q_{2}\right\|=\left\|\left(q_{1} \odot \xi\right)-\left(q_{2} \odot \xi\right)\right\|, \forall \xi \in \Xi$.

- Given a TSRVF $q$ and an initial trajectory point, one can reconstruct the corresponding path $\beta$, such that $Q(\beta)=$ $q$, by solving an ordinary differential equation (ODE), as discussed in [36].

As we will see next, these properties enable efficient temporal registration of trajectories and subsequent rate-invariant statistical analysis. In what follows, let $\mathcal{Q}$ denote the space of TSRVFs equipped with the $\mathbb{L}^{2}$ metric defined in Eqn. 7

\subsubsection{Temporal registration}

Under the TSRVF representation, the temporal registration problem in Eqn. (5), which involved optimization over $\Xi$, can now be reformulated using the standard $\mathbb{L}^{2}$ metric on the space of TSRVFs:

$$
\xi^{*}=\underset{\xi \in \Xi}{\operatorname{argmin}}\left\|q_{1}-q_{2} \odot \xi\right\| .
$$

This problem can be solved efficiently using a Dynamic Programming algorithm [36], [37]. Then, the rate-invariant distance between two trajectories is given by:

$$
d\left(\beta_{1}, \beta_{2}\right)=\inf _{\xi \in \Xi}\left\|q_{1}-q_{2}, \odot \xi\right\| .
$$




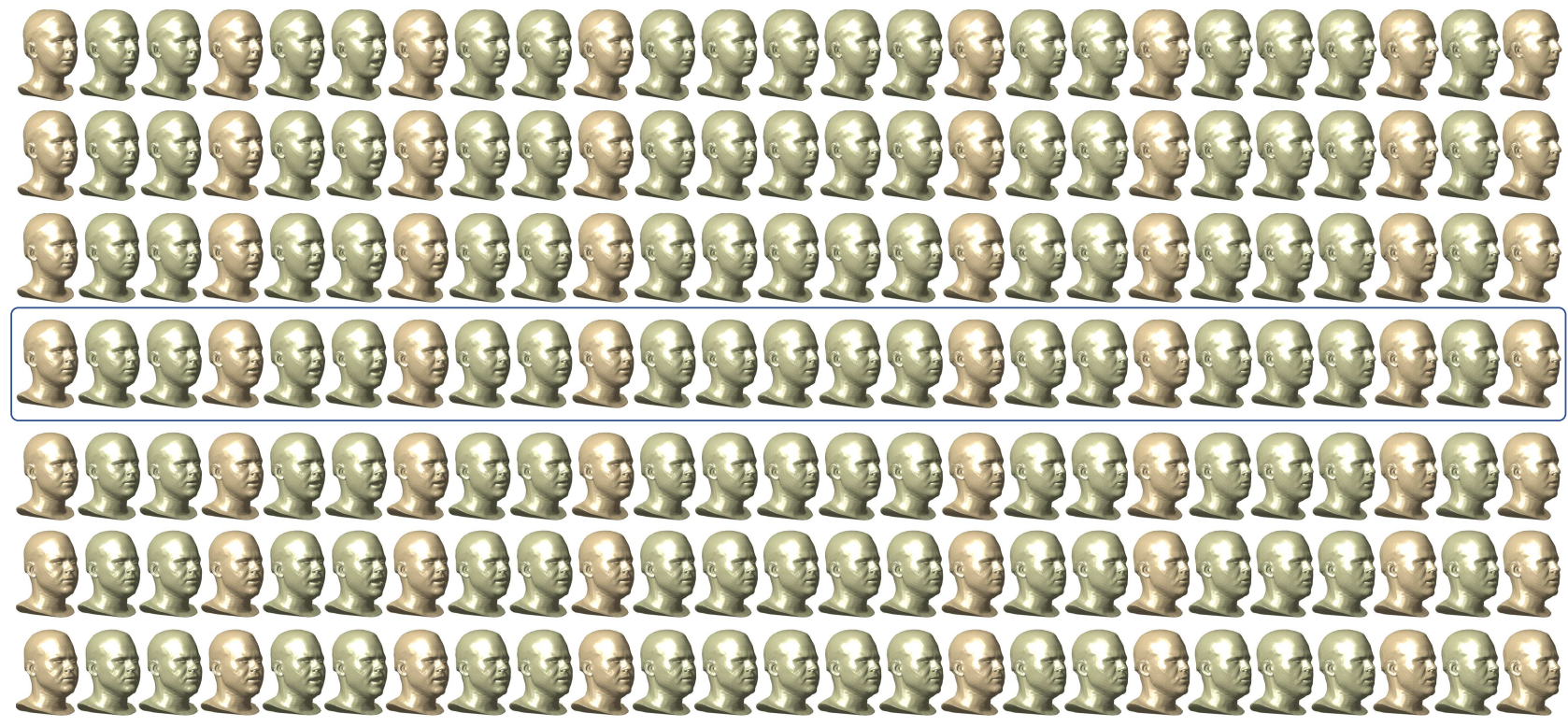

Fig. 4. Example of a geodesic between the source 4D surface (top row) and the target 4D surface (bottom row) after spatiotemporal registration. The highlighted row corresponds to the mean 4D surface. A video of the figure is included in the Supplementary Material.

\subsubsection{Geodesics between 4D surfaces}

We now summarize our contributions so far and the entire pipeline. Let $\alpha_{1}, \alpha_{2} \in \mathcal{M}_{\mathcal{F}}$ be two $4 \mathrm{D}$ surfaces. The pipeline to spatiotemporally register them, and to compute the geodesic path between them, can be summarized as follows.

(1) Proposed spatial registration. The goal is to spatially register the surfaces in $\alpha_{1}$ and $\alpha_{2}$ to the same reference surface. In general, the reference surface can be any arbitrary surface. However, for simplicity, we choose it to be $\alpha_{1}(0)$, i.e., the first surface in the sequence $\alpha_{1}$. The spatial registration can then be performed in two steps.

- Compute the SRNF maps, $\forall t \in[0,1], \beta_{1}(t)=H\left(\alpha_{1}(t)\right)$ and $\beta_{2}(t)=H\left(\alpha_{2}(t)\right)$.

- Spatially register $\beta_{1}$ and $\beta_{2}$, and as a result $\alpha_{1}$ and $\alpha_{2}$, to the reference surface, using the algorithm described in Section 3.1.2

For the simplicity of notation, we also use $\beta_{1}$ and $\beta_{2}$ to denote the spatially-registered trajectories.

(2) Proposed temporal alignment. Now, $\beta_{1}$ and $\beta_{2}$ are elements of $\mathcal{M}_{h}$; see Fig. 3 We perform temporal registration in 3 steps.

- Map $\beta_{1}$ and $\beta_{2}$ to the TSRVF space $\mathcal{Q}: q_{1}=Q\left(\beta_{1}\right)$ and $q_{2}=Q\left(\beta_{2}\right)$. Now, $q_{1}$ and $q_{2}$ are elements of $\mathcal{Q}$.

- Find $\xi^{*}$, the optimal reparametrization that registers $q_{2}$ to $q_{1}$ by solving Eqn. (8).

- $q_{2}^{*} \leftarrow q_{2} \odot \xi^{*}$ and $\beta_{2}^{*} \leftarrow \beta_{2} \circ \xi^{*}$.

(3) Proposed geodesic computation. Since $\mathcal{Q}$ is Euclidean, the geodesic path $\Lambda_{q}$ between $q_{1}$ and $q_{2}^{*}$ is a straight line:

$$
\Lambda_{q}(\tau)=(1-\tau) q_{1}+\tau q_{2}^{*}, \tau \in[0,1] .
$$

Next, we map $\Lambda_{q}$ back to $\mathcal{M}_{h}$ using the inverse TSRVF map, i.e., $\forall \tau, \Lambda_{\beta}(\tau)=Q^{-1}\left(\Lambda_{q}(\tau)\right)$. The computation of the inverse mapping uses the starting point on the trajectory and has a closed-form solution, making it computationally efficient. This is described in detail in [36]. After applying the inverse mapping to the entire geodesic path, we have $\Lambda_{\beta}(0)=\beta_{1}, \Lambda_{\beta}(1)=\beta_{2}$, and $\beta_{\tau}=\Lambda_{\beta}(\tau), \tau \in(0,1)$, i.e., a geodesic path between the SRNF curves $\beta_{1}$ and $\beta_{2}$.

(4) Visualization. To visualize geodesic paths between $4 \mathrm{D}$ surfaces (and not their SRNFs), we need to further map all SRNFs on the trajectory $\Lambda_{\beta}(\tau)$ to their corresponding surfaces in $\mathcal{F}$. This is done using the inverse SRNF map, i.e., $\forall \tau \in[0,1], t \in[0,1], \Lambda(\tau)(t)=H^{-1}\left(\Lambda_{\beta}(\tau)(t)\right)$. Unlike the TSRVF map whose inverse can be computed analytically, inversion of the SRNF map, whose injectivity and surjectivity are yet to be determined, has to be accomplished numerically. Fortunately, Laga et al. [4] showed that, for a given SRNF of a valid surface, one can always retrieve the original surface, up to translation, using an efficient, iterative numerical inversion procedure. We use this approach in this paper.

Now, $\Lambda$ is the geodesic path between the $4 \mathrm{D}$ surfaces $\alpha_{1}$ and $\alpha_{2}^{*}$, i.e., $\Lambda(0)=\alpha_{1}, \Lambda(1)=\alpha_{2}^{*}$, and $\alpha_{\tau}=\Lambda(\tau)$ is a $4 \mathrm{D}$ surface at time $\tau$ along the geodesic path. Fig. 4 shows an example of a geodesic between two 4D surfaces representing talking faces. Each row correspond to one $4 \mathrm{D}$ surface. The top is the source $4 \mathrm{D}$ surface, the bottom row is the target $4 \mathrm{D}$ surface, after optimal spatiotemporal registration, and the highlighted row in the middle corresponds to the mean $4 \mathrm{D}$ surface. The temporal registration is further illustrated in Fig. 5. where we show the source $4 \mathrm{D}$ surface, the target $4 \mathrm{D}$ surface before spation-temporal registration, and the target $4 \mathrm{D}$ surface before spation-temporal registration. Section 5 provides more examples of geodesics computed between various types of $4 \mathrm{D}$ surfaces.

\section{Statistical ANALYSIS OF 4D SURFACES}

Now that we have devised all of the required mathematical tools for comparing $4 \mathrm{D}$ surfaces, we shift our focus to how these tools can be used to build a $4 \mathrm{D}$ atlas from a sample 


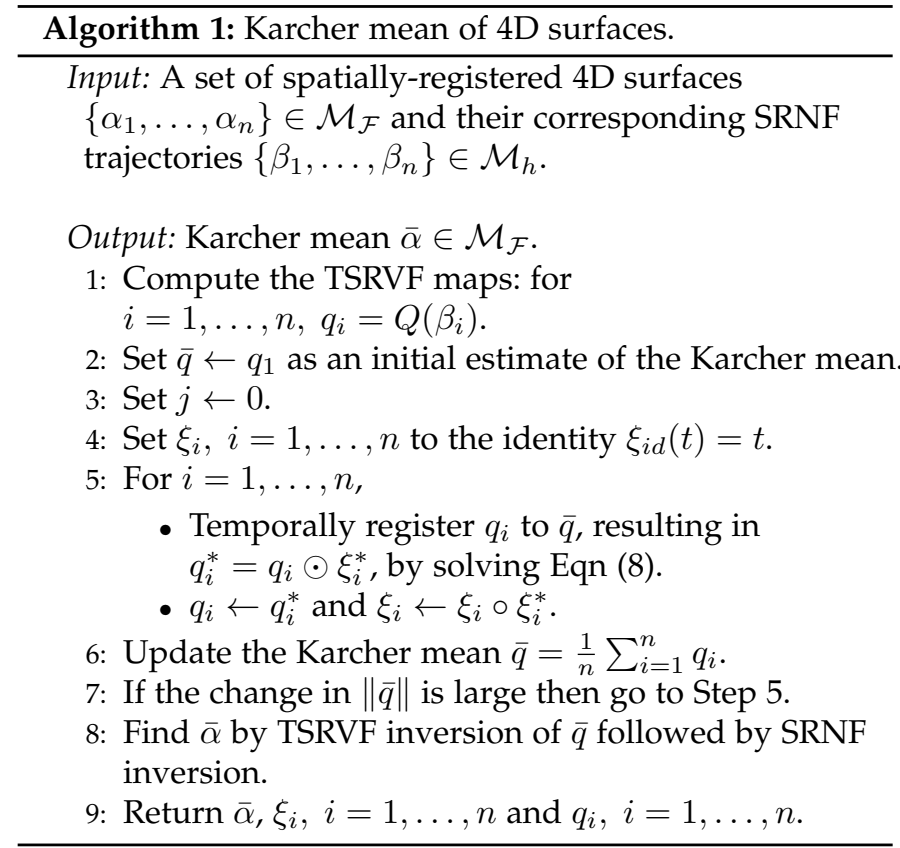

of $4 \mathrm{D}$ surfaces. Let $\alpha_{1}, \cdots, \alpha_{n}$ be a set of $4 \mathrm{D}$ surfaces and $\beta_{1}, \cdots, \beta_{n}$ be their corresponding trajectories in $\mathcal{C}_{h}$. Here, we also assume that all of the surfaces, and their corresponding SRNFs, have already been spatially registered to a common reference, as outlined in Section 3.1.2 We proceed to map all of the $4 \mathrm{D}$ surfaces to their corresponding TSRVFs, hereinafter denoted by $q_{1}, \cdots, q_{n}$, and compute statistics in the space of TSRVFs. As before, all results are mapped at the end to the original space of surfaces $\mathcal{F}$ for visualization. We show in this section how to use this framework to compute means and modes of variation, and how to synthesize novel 4D surfaces by sampling from probability distrbutions fitted to a collection of exemplar 4D surfaces.

Mean of 4D surfaces. Intuitively, the mean of a collection of $4 \mathrm{D}$ surfaces is the $4 \mathrm{D}$ surface that is as close as possible to all of the 4D surfaces in the collection, under the specified distance measure (or metric). This is known as the Karcher mean and is defined as the $4 \mathrm{D}$ surface that minimizes the sum of squared distances to all of the $4 \mathrm{D}$ surfaces in the given sample. In other words, we seek to solve the following optimization problem, defined in the space of TSRVFs:

$$
\bar{q}=\underset{q \in \mathcal{Q}}{\operatorname{argmin}} \sum_{i=1}^{n} \min _{\xi_{i} \in \Xi}\left\|q-q_{i} \odot \xi_{i}\right\|^{2} .
$$

Algorithm 1 describes the proposed procedure for solving this optimisation problem. It outputs the TSRVF Karcher mean $\bar{q}$, the optimal temporal reparameterizations $\xi_{i}^{*}, i=$ $1, \ldots, n$, and the temporally registered TSRVFs $q_{i}^{*}=q_{i} \odot \xi_{i}^{*}$; again, for simplified notation we simply use $\xi_{i}$ and $q_{i}$ to denote the optimal temporal reparameterizations and the temporally registered TSRVFs. The mean $4 \mathrm{D}$ surface can be obtained by mapping the mean TSRVF using TSRVF inversion followed by SRNF inversion [4].

Principal modes of variation. Since the TSRVF space is Euclidean, the principal modes of variation can also be computed in a standard way, i.e., using the Singular Value
Decomposition (SVD) of the covariance matrix. In the following, we assume that the TSRVFs are sampled using a finite set of points and appropriately vectorized. Let $K=\frac{1}{n-1}\left(q_{i}-\bar{q}\right)\left(q_{i}-\bar{q}\right)^{\top}$ be the covariance matrix of the input sample, $\sigma_{i}, i=1, \ldots, k$ its $k$-leading eigenvalues, and $\Sigma_{i}, i=1, \ldots, k$ the corresponding eigenvectors. Then, one can explore the variability in the $i$-th principal direction using $q_{\tau}=\bar{q}+\tau \sqrt{\sigma_{i}} \Sigma_{i}$, where $\tau \in \mathbb{R}$. To visualize this principal direction of variation, we again use TSRVF inversion followed by SRNF inversion to compute the 4D surface $\alpha_{\tau}$, such that $Q\left(H\left(\alpha_{\tau}\right)\right)=\bar{q}+\tau \sqrt{\sigma_{i}} \Sigma_{i}, \tau \in \mathbb{R}$.

Random 4D surface synthesis. Given the mean and the $k$ leading principal modes of variation, any TSRVF $q$ of a $4 \mathrm{D}$ surface $\alpha$ can be represented in a parameterized form as:

$$
q=\bar{q}+\sum_{i=1}^{k} \tau_{i} \sqrt{\sigma_{i}} \Sigma_{i}, \tau_{i} \in \mathbb{R} .
$$

Thus, to generate a random TSRVF, we only need to generate $k$ random values $\tau_{i} \in \mathbb{R}$ and plug them into Eqn. (12). Then, to compute the corresponding random $4 \mathrm{D}$ surface, we apply the inverse TSRVF map followed by the inverse SRNF map. Also, by enforcing each $\tau_{i}$ to be within a certain range, e.g., $[-1,1]$, we can ensure that the generated random $4 \mathrm{D}$ surfaces are similar to the given sample and thus plausible.

This procedure allows to generate new random $4 \mathrm{D}$ surfaces. However, it does not offer any control over the generation process, which is completely random. In many situations, we would like to control this process using a set of parameters. For instance, when dealing with $4 \mathrm{D}$ facial expressions, these parameters can be the degree of sadness, how fat or skinny the face is, etc. This type of control can be efficiently implemented using regression in the TSRVF space, a problem that we plan to explore in the future.

\section{Results}

In this section, we show some results of the proposed framework. Section 5.1 focuses on spatiotemporal registration and geodesic computation between $4 \mathrm{D}$ surfaces. Section 5.2 focuses on the computation of statistical summaries such as means and modes of variation, while Section 5.3 shows examples of $4 \mathrm{D}$ surfaces randomly synthesized using the proposed statistical analysis framework. We use three types of datasets: (1) The VOCA dataset [6], which contains 4D facial scans, captured at $60 \mathrm{fps}$, of 12 subjects speaking various sentences. (2) The MPI 4D FAUST dataset [7], which contains high-resolution 4D scans of 10 human subjects in motion, captured at $60 \mathrm{fps}$. In total, the dataset contains 129 dynamic performances, and (3) The MPI 4D CAPE dataset [8], which contains high-resolution 4D scans of 10 male and 5 female subjects in clothing. These datasets come as polygonal meshes, with consistent triangulation and given registration across all the meshes. We spherically parameterize these surfaces using Kurtek et al. [17]'s implementation of the spherical parameterization approach of [38]. We also apply randomly-generated spatial diffeomorphisms to simulate non-registered surfaces. Our framework does not use, either explicitly or implicitly, the provided vertex-wise correspondences 


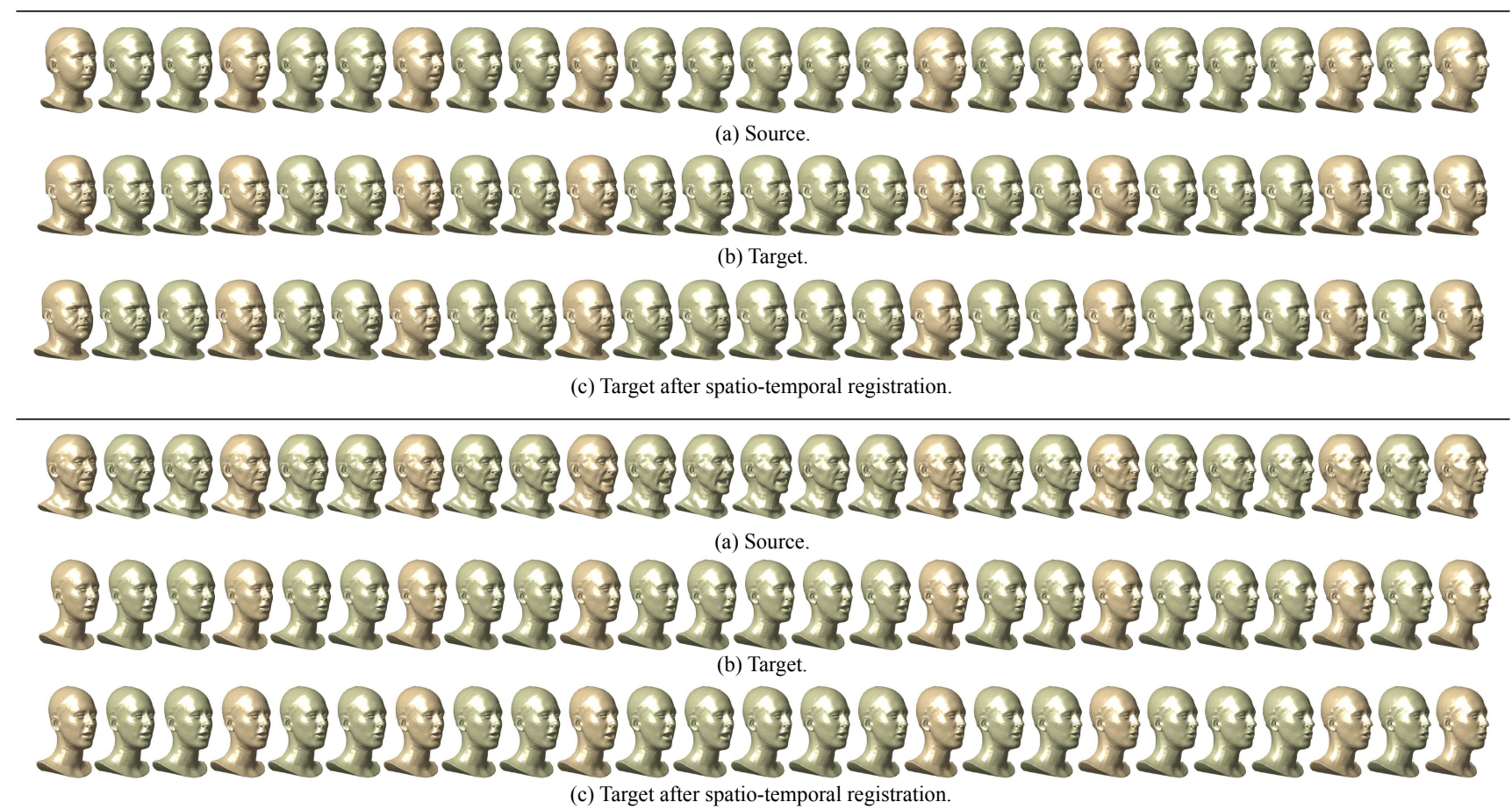

(c) Target after spatio-temporal registration.

Fig. 5. Examples of the spatiotemporal registration of two facial expressions (4D faces). In each example, we show (a) the source 4D face, (b) the target $4 \mathrm{D}$ face, and (c) the target $4 \mathrm{D}$ face after spatiotemporal registration using the proposed framework. Note how the spatiotemporally registered target 4D surface became fully synchronised with the source 4D surface. The full video sequence is provided in the supplementary material.

\subsection{Spatio-temporal registration and $4 \mathrm{D}$ geodesics}

In this section, we consider pairs of $4 \mathrm{D}$ facial expressions from the VOCA dataset. We first reparameterize each $4 \mathrm{D}$ surface using randomly-generated time-warping functions to simulate facial expressions performed at different execution rates. We then apply the framework proposed in this article to spatiotemporally register them. Figs. 5 shows two examples of such spatiotemporal registration. In each example, we show (a) the source $4 \mathrm{D}$ surface, (b) the target $4 \mathrm{D}$ surface before spatiotemporal registration, and (c) the target 4D surface after spatiotemporally registering it onto the source. We also highlight some key frames. As one can see, the original 4D surfaces are not temporally aligned, i.e., they significantly differ in their execution rates. The spatiotemporal registration framework synchronizes the source and target expressions, thus enabling their comparison, interpolation and averaging. We also perform a similar experiment on the human body shapes of the FAUST [7] and CAPE [8] datasets; see Figs. 6. 7, and 9(a)-(c). Compared to faces, human body shapes are very challenging to analyse since they perform complex articulated motions, which result in large bending and stretching of their surfaces.

4D geodesics. Fig. 8, shows geodesics between $4 \mathrm{D}$ human body shapes. In this example, both the source and target perform a punching action but at different rates. We show the geodesic before temporal registration and the geodesic after the spatiotemporal registration of the target $4 \mathrm{D}$ surface onto the source. Unlike the jumping action of Fig. 6 , the left hand of the target surface does not perform the same action as the left hand of the source surface. Yet, our framework is able to bring these two $4 \mathrm{D}$ surfaces as close as possible to each others. The supplementary materials includes a video of the sequence and more examples of geodesics between: 4D faces (from VOCA), 4D human bodies (from DFAUST), and clothed 4D human bodies (from CAPE).

Ablation study. We undertake an ablation study to demonstrate the utility of the various components of the proposed pipeline. Using the human body shapes, which are the most challenging since they undergo a large articulated motion, we show the mean 4D surface obtained: (1) with the SRNF representation, with spatial registration, and with temporal registration, (2) with the SRNF representation, with spatial registration, and without temporal registration, (3) without SRNF representation, with spatial registration, and with temporal registration, and (4) without SRNF representation, with spatial registration, and without temporal registration. The last two cases are equivalent to the linear interpolation in the original surface space, after spatial registration. In all cases, we perform the spatial registration using the SRNF framework, which has been already evaluated and compared to previous techniques in [4].

Fig. 9 shows some sample results. First, we can see that the temporally-aligned target $4 \mathrm{D}$ surface (Fig. 9 (c)) is very close to the source 4D surface of Fig. 9. (a). We observe that the right hands became fully synchronised. As such, the mean $4 \mathrm{D}$ surface obtained after temporal registration (Fig. 9.(e)) became fully synchronised with the source and the aligned target, unlike the mean $4 \mathrm{D}$ surface in Fig. 9 . (d), which has been obtained without temporal registration. Second, in the mean $4 \mathrm{D}$ surfaces obtained without the SRNF framework (Figs. 9f(f) and (g)), i.e., obtained through linear interpolation in the original space of surfaces, we can observe that the parts that undergo large articulated 


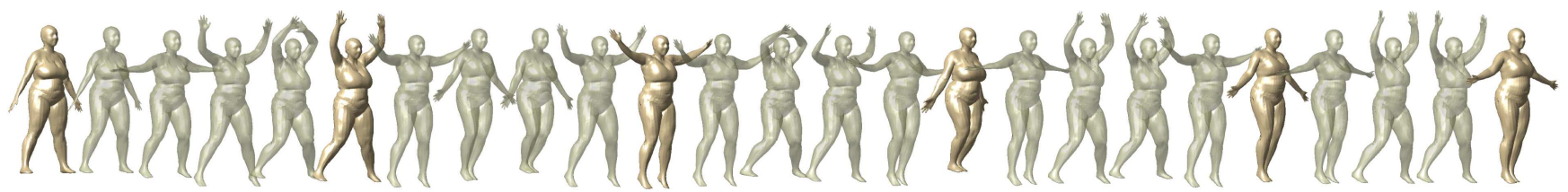

(a) Source.

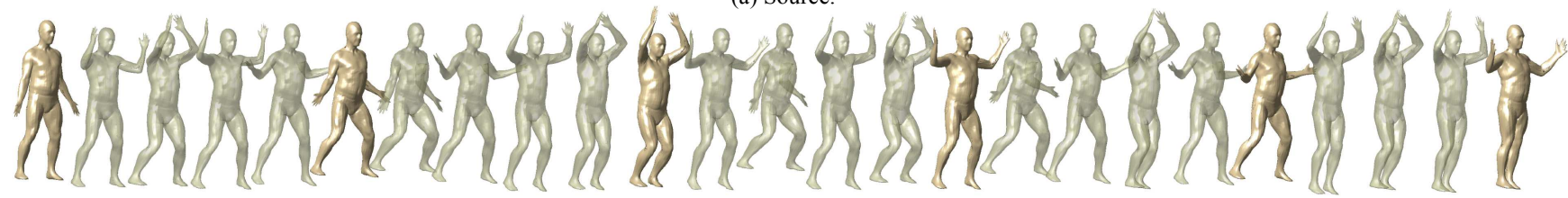

(b) Target before spatio-temporal registration.

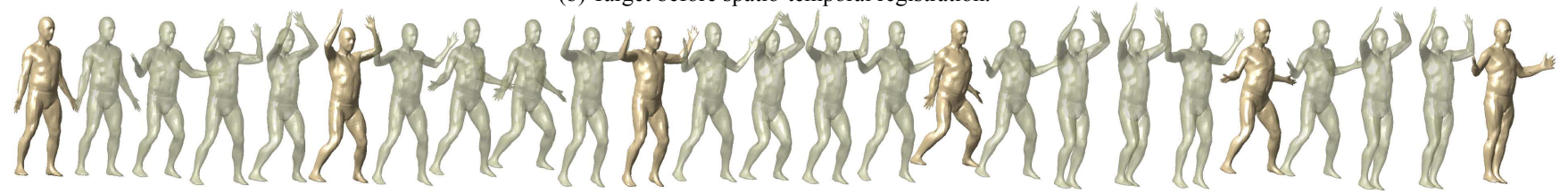

(c) Target after spatio-temporal registration.

Fig. 6. Example of the spatiotemporal registration, using the proposed algorithm, of two 4D human body shapes (from the FAUST dataset) performing a jumping action at different speeds. Note how the spatiotemporally registered target 4D surface in (c) became synchronised with the source 4D surface in (a). The full video sequence is provided in the supplementary material.

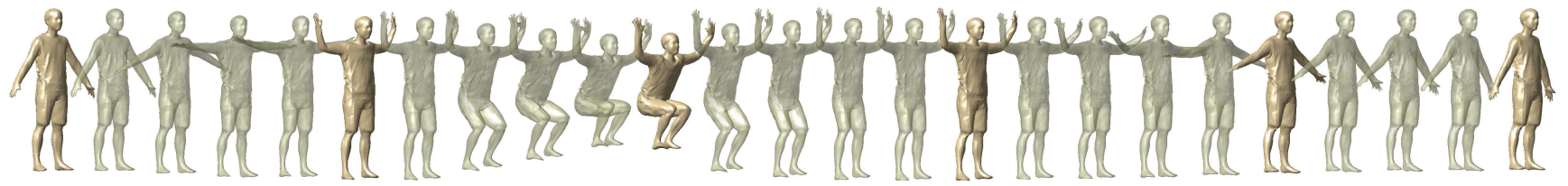

(a) Source.

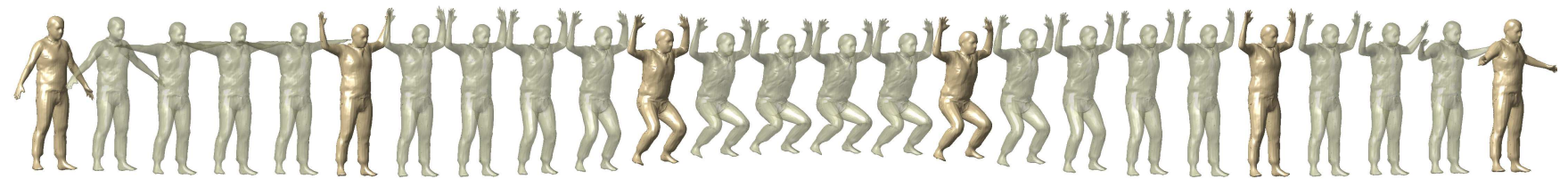

(b) Target before spatio-temporal registration.

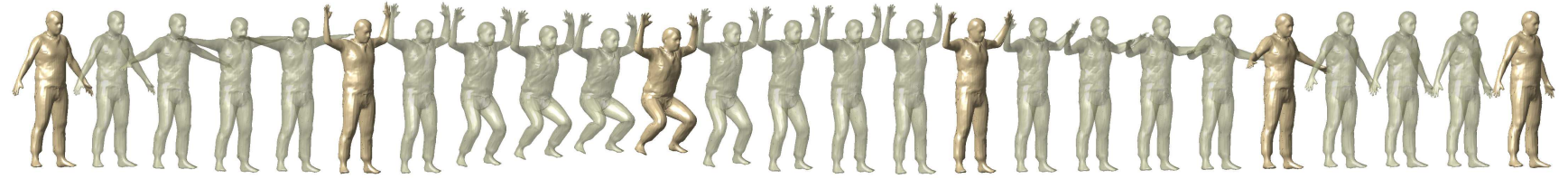

(c) Target after spatio-temporal registration.

Fig. 7. Example of the spatiotemporal registration, using the proposed algorithm, of two 4D body shapes with different clothing from the CAPE dataset). Note how the spatiotemporally registered target 4D surface in (c) became fully synchronised with the source 4D surface in (a). The full video sequence is provided in the supplementary material.

motion (e.g., the arms) unnaturally shrink. This shrinkage is stronger in Fig. 9-(f) since the mean is obtained without temporal registration. Fig. 10 shows a zoom out on the two time frames indicated with a blue arrow in Fig. 9 .

Evaluation of the spatiotemporal registration. To quantitatively evaluate the performance of the proposed spatiotemporal registration, we first consider a 4D surface $\alpha$, parameterize it with randomly generated diffeomorphims $\xi_{i}, i \in\{1, \cdots, 100\}$, to obtain new $4 \mathrm{D}$ surfaces $\alpha_{i}=\alpha \circ \xi_{i}$. Next, we compute the distances $d_{i}, i=1, \ldots, 100$, between $\alpha$ and $\alpha_{i}$ using the expression given in Eqn. (7). We then plot the boxplots of the resulting distances for 12 sequences from the VOCA dataset in Fig. 11-(a). Ideally, $\forall i, d_{i}=0$. However, since the $4 \mathrm{D}$ surfaces are not spatiotemporally registered, the distances are large in most cases.

Next, we compute the optimal spatiotemporal registration, i.e., for every $4 \mathrm{D}$ surface $\alpha_{i}$, we find the optimal diffeomorphism $\xi_{i}^{*}$ that aligns $\alpha_{i}$ onto $\alpha$. Let $\alpha_{i}^{*}=\alpha_{i} \circ \xi_{i}^{*}$. Ideally, the distance between $\alpha_{i}^{*}$ and $\alpha$ computed using Eqn. (7) should be very small. Fig. 11-(b) shows the boxplots of the distances between $\alpha_{i}^{*}$ and $\alpha$ for the same 12 sequences from the VOCA dataset. Compared to the distances between the original unregistered 4D expressions shown in Fig. 11. (a), these distances are significantly lower.

Computation time. Our approach runs fully on CPU. The Matlab implementation of the spatiotemporal registration process takes less than 31.43 seconds on $4.2 \mathrm{GHz}$ Intel Core i7 with 32 GB of RAM. The visualization process, however, 

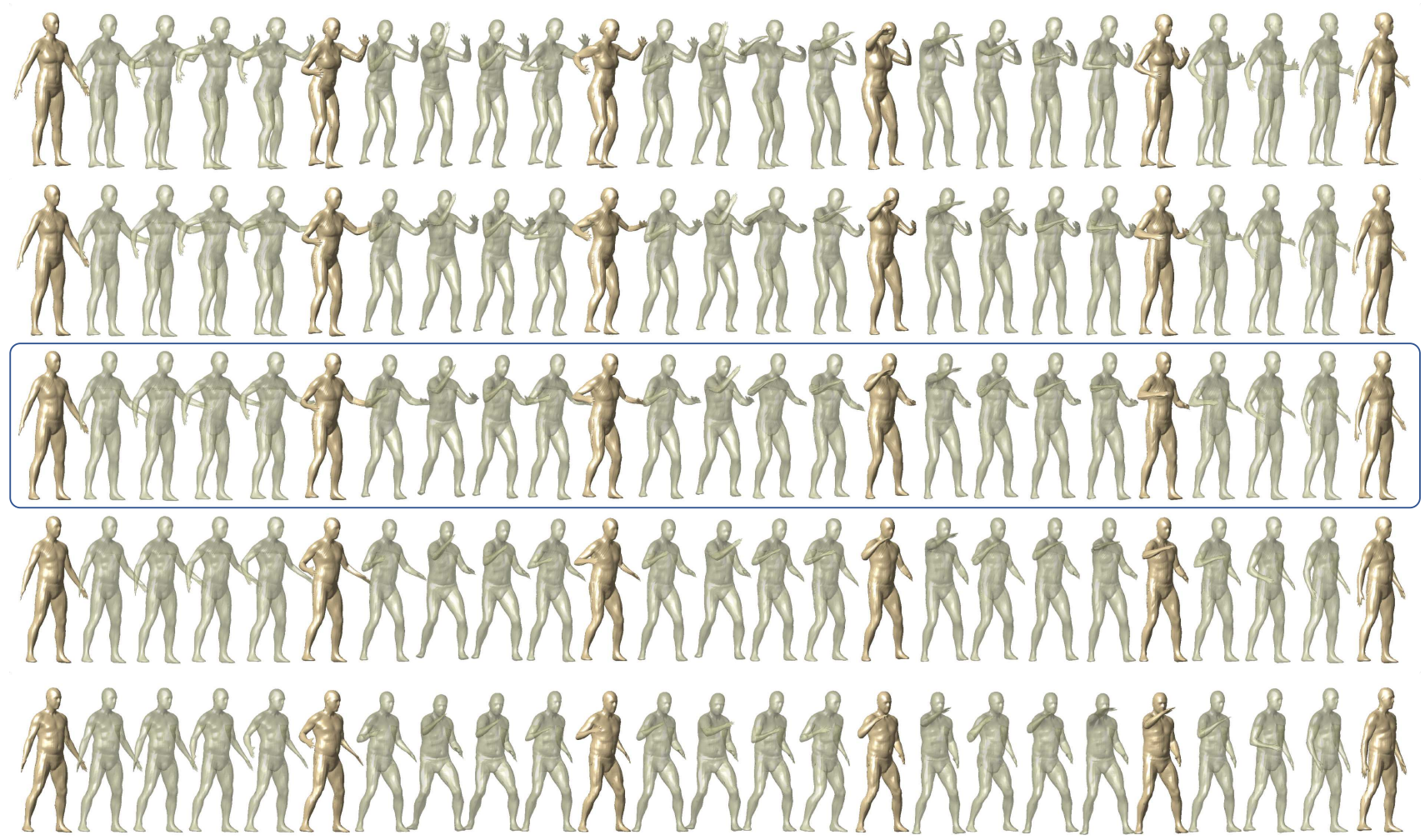

(a) Before registration. The highlighted middle row corresponds to the mean 4D surface.
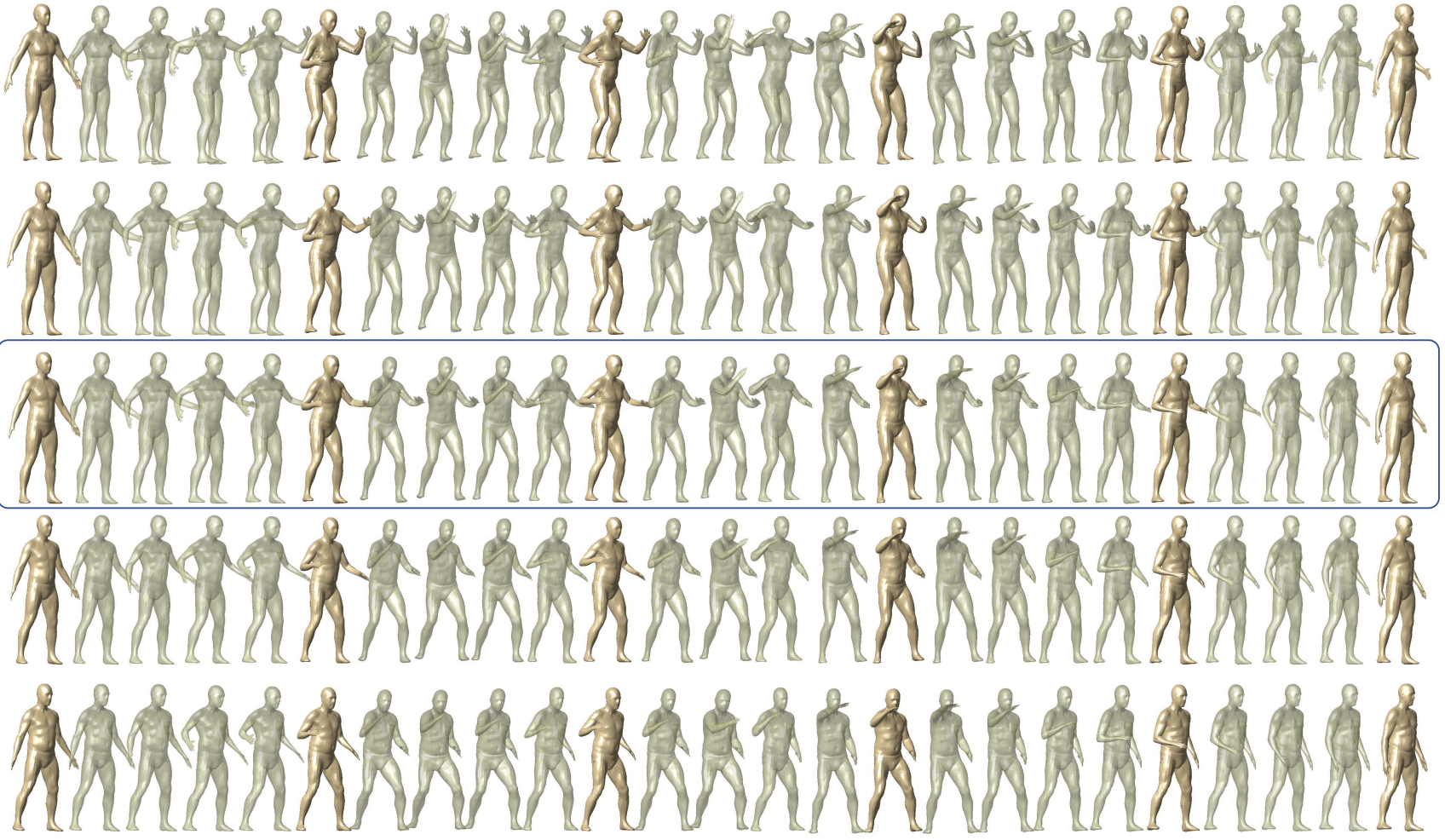

(b) After registration. The highlighted middle row corresponds to the mean 4D surface.

Fig. 8. Example of a geodesic between 4D surfaces corresponding to punching actions ((a) before registration, and (b) after registration). In each example, we show in each row the source 4D surface, the target 4D surface, and three intermediate 4D surfaces along the geodesic between the source and the target. Observe how misaligned are the highlighted frames before registration. A video illustrating these sequences is included in the Supplementary Material.

which is needed when computing geodesics, means, and modes of variation, and when synthesizing random $4 \mathrm{D}$ surfaces, relies on the inversion of the SRNF maps. This requires 6 seconds per frame and a total of 30 mins for the 


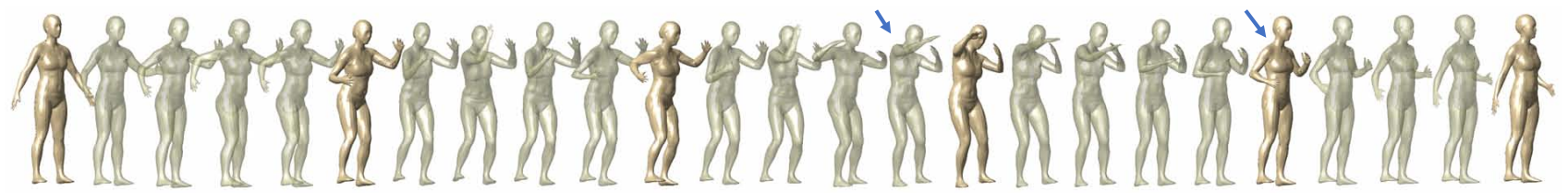

(a) Source.

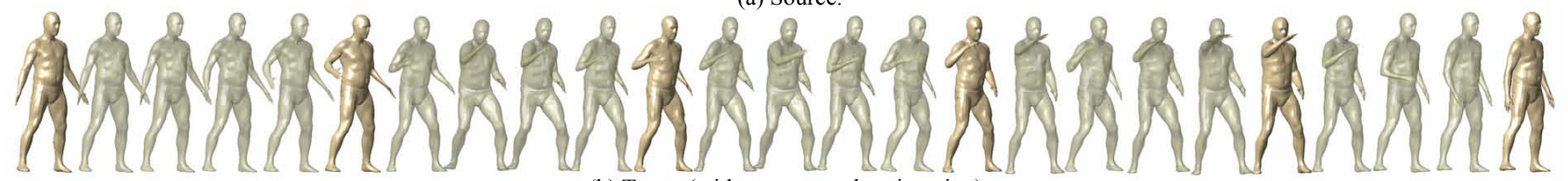

(b) Target (with no temporal registration).

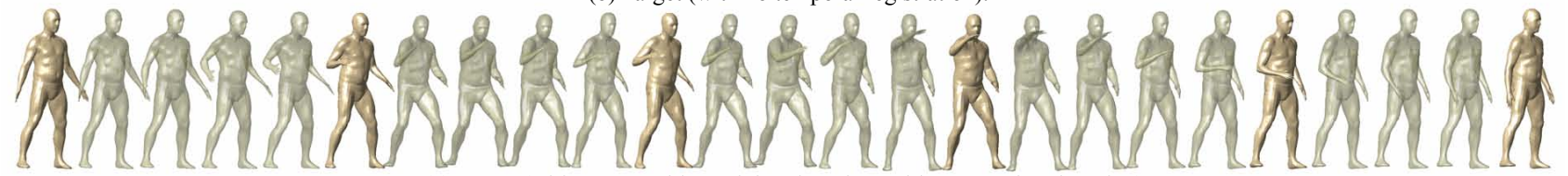

(c) Target (with SRNF, with spatial registration, with temporal registration)

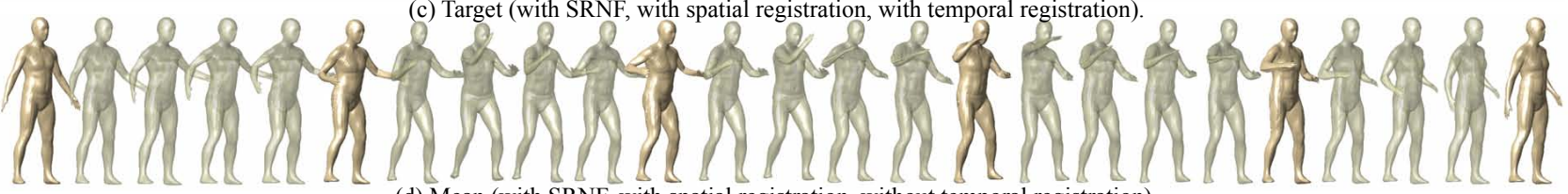

(d) Mean (with SRNF, with spatial registration, without temporal registration).

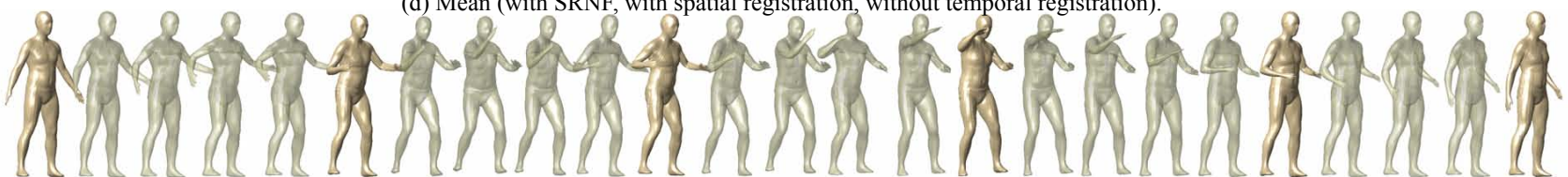

(e) Mean (with SRNF, with spatial registration, with temporal registration).

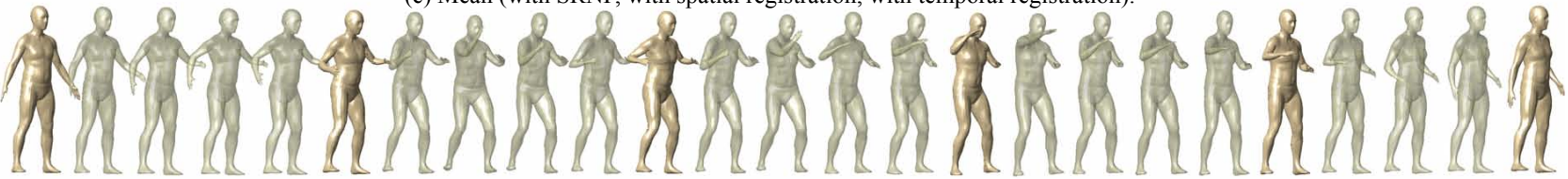

(f) Mean (without SRNF, with spatial registration, without temporal registration).

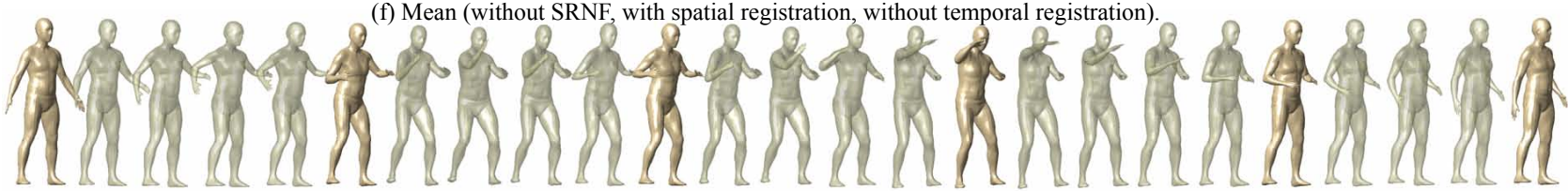

(g) Mean (without SRNF, with spatial registration, with temporal registration).

Fig. 9. Ablation study: illustration of the effect of the different components of the proposed framework on the quality of the mean 4D surface, which is the middle point along the geodesic between the source and target 4D surfaces. The 4D surfaces are from the FAUST dataset. Please refer to Fig. 10 for a zoom on the temporal frames indicated with an arrow. A video illustrating these sequences is included in the Supplementary Material.

300 temporal frames used in this paper. All the experiments were undertaken using high spatial spherical resolution $(256 \times 256)$.

\subsection{Summary statistics}

Next, we consider a set of unregistered 4D surfaces and compute their mean and principal modes of variation. Fig. 12 shows the 4D mean computed from six 4D human shapes performing different types of actions (jumping and punching). The figure also shows the input 4D surfaces after their spatiotemporal registration (please refer to the video in the supplementary material for an illustration of the input $4 \mathrm{D}$ surfaces before spatiotemporal registration). As we can see, despite the large articulated motion, the large differences in the type of actions, and the large differences in the execution rates of the $4 \mathrm{D}$ surfaces, our framework is able to co-register them and generate a plausible average 4D surface. Figs. 13,a) and (b) show the first two principal modes of variation computed on input 4D facial surfaces. Please refer to the videos in the supplementary material, which includes the input $4 \mathrm{D}$ surfaces as well as additional additional results. As we can see, the proposed procedure is able to co-register the $4 \mathrm{D}$ surfaces even in the presence of large elastic deformations and large variations in the execution rates of the $4 \mathrm{D}$ surfaces. The computed mean also captures the main features of the dataset. The principal modes of variation further capture relevant variability in the given data.

\subsection{D surface synthesis}

Finally, Fig. 13-(c) shows five 4D facial expressions randomly sampled from the Gaussian distribution with parameters estimated using the VOCA dataset. To ensure that the synthesized 4D surfaces are plausible, we only consider those that are within two standard deviations along each principal mode of variation. We refer the reader to the 


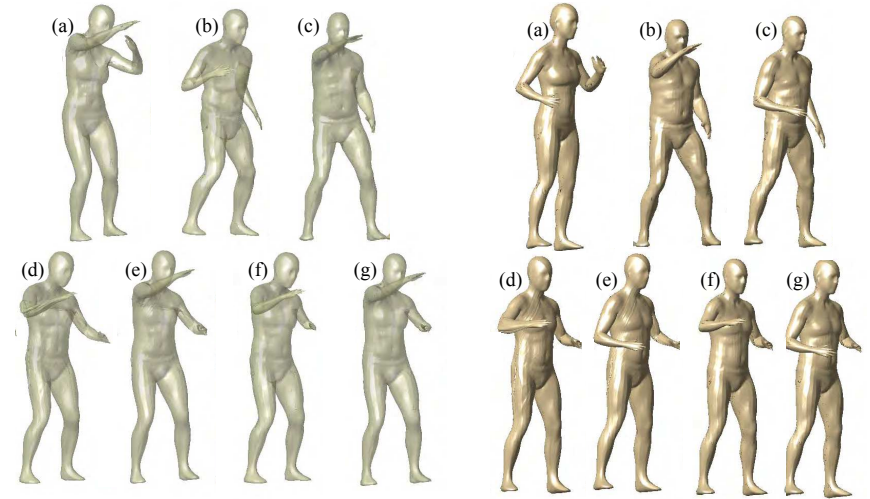

Fig. 10. Zoom on the two frames indicated with a blue arrow in Fig. 9 Refer to Fig. 9 for the meaning of the labels (a) to (g). Observe in (f) and (g), which use linear interpolation, how the left arm is shrunk compared to $(e)$, which has been obtained using the proposed framework.

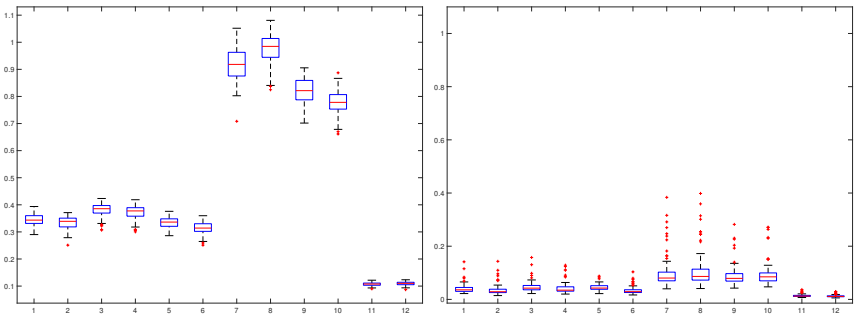

(a)

(b)

Fig. 11. Boxplots of distances between (a) unregistered 4D surfaces generated using 100 random diffeomorphic transformations of a single 4D surface for 12 sequences from the VOCA dataset, and (b) spatiotemporal registered surfaces.

supplementary material for videos of all of the randomly generated $4 \mathrm{D}$ surfaces. The ability to synthesize novel $4 \mathrm{D}$ surfaces can benefit many applications in computer vision and graphics. In particular, it can be used to augment datasets for efficient training of deep learning models.

\section{CONCLUSION}

We proposed a new framework for the statistical analysis of longitudinal 3D shape data (or $4 \mathrm{D}$ surfaces), i.e., surfaces that deform over time, e.g., 3D human body shapes performing actions at different execution rates or 3D human faces pronouncing sentences at different speeds. Unlike traditional techniques, which only consider how features such as landmarks or measurements vary over time, our framework considers the deformation of the entire surface of a 3D object. Our key contribution is in the representation of $4 \mathrm{D}$ surfaces as trajectories in the space of SRNFs, and the use of Square Root Velocity Functions to statistically analyze such trajectories. The proposed framework is able to spatiotemporally register $4 \mathrm{D}$ surfaces, even in the presence of large elastic deformations and large variations in the execution rates of the expressions. It is also able to compute geodesics and summary statistics, which in turn can be used to randomly synthesize new, unseen $4 \mathrm{D}$ surfaces.

Although we only demonstrated the proposed $4 \mathrm{D}$ analysis framework on human body shapes and facial surfaces, it is general and can be applied to other types of surfaces. Our current implementation, however, is limited to surfaces that are homeomorphic to a sphere or a disk. We plan, in the future, to extend the framework to higher genus surfaces by exploring different parameterization methods. The framework is also limited to surfaces that bend and stretch but do not change in topology. As such, it cannot be applied to tree-like shapes, e.g., botanical trees or roots. However, the concept of representing deformations as trajectory in a shape space can also be extended to tree-shape spaces such as those proposed in [41], [42].

The proposed framework has various applications in computer vision, graphics, biology, and medicine. For instance, in computer vision, collecting large animations (i.e., $4 \mathrm{D}$ sequences) to train deep neural networks, e.g., for 3D reconstruction of action recognition [43], [44], is complex and time consuming. The proposed framework can contribute to solving this problem by automatically synthesizing new samples from a small dataset. The proposed framework can also be used to statistically analyze how anatomical organs deform as a result of natural growth or disease progression. We plan to explore these applications in the future.

\section{REFERENCES}

[1] P. Werner, D. Lopez-Martinez, S. Walter, A. Al-Hamadi, S. Gruss, and R. Picard, "Automatic recognition methods supporting pain assessment: A survey," IEEE Transa. Affective Computing, 2019.

[2] B. Egger, W. A. Smith, A. Tewari, S. Wuhrer, M. Zollhoefer, T. Beeler, F. Bernard, T. Bolkart, A. Kortylewski, S. Romdhani et al. "3d morphable face modelspast, present, and future," ACM TOG, vol. 39, no. 5, pp. 1-38, 2020.

[3] B. Allen, B. Curless, and Z. Popović, "The space of human body shapes: Reconstruction and parameterization from range scans," ACM Transactions on Graphics, vol. 22, no. 3, pp. 587-594, Jul. 2003.

[4] H. Laga, Q. Xie, I. H. Jermyn, and A. Srivastava, "Numerical inversion of SRNF maps for elastic shape analysis of genus-zero surfaces," IEEE PAMI, 2017.

[5] I. Jermyn, S. Kurtek, E. Klassen, and A. Srivastava, "Elastic shape matching of parameterized surfaces using square root normal fields," IEEE European Conference on Computer Vision „, vol. 5, no. 14, pp. 805-817, 2012.

[6] D. Cudeiro, T. Bolkart, C. Laidlaw, A. Ranjan, and M. Black "Capture, learning, and synthesis of 3D speaking styles," IEEE CVPR, pp. 10101-10111, 2019. [Online]. Available: http://voca.is.tue.mpg.de/

[7] F. Bogo, J. Romero, G. Pons-Moll, and M. J. Black, "Dynamic FAUST: Registering human bodies in motion," in IEEE CVPR, Jul. 2017.

[8] Q. Ma, J. Yang, A. Ranjan, S. Pujades, G. Pons-Moll, S. Tang, and M. J. Black, "Learning to dress 3d people in generative clothing," in IEEE CVPR, 2020, pp. 6469-6478.

[9] T. F. Cootes, C. J. Taylor, D. H. Cooper, and J. Graham, "Active shape models-their training and application," Computer vision and image understanding, vol. 61, no. 1, pp. 38-59, 1995.

[10] V. Blanz and T. Vetter, "A morphable model for the synthesis of $3 \mathrm{~d}$ faces," in ACM Siggraph, 1999, pp. 187-194.

[11] D. Anguelov, P. Srinivasan, D. Koller, S. Thrun, J. Rodgers, and J. Davis, "Scape: shape completion and animation of people," in ACM SIGGRAPH 2005 Papers, 2005, pp. 408-416.

[12] N. Hasler, C. Stoll, M. Sunkel, B. Rosenhahn, and H.-P. Seidel, “A statistical model of human pose and body shape," in CGF, vol. 28, no. 2, 2009, pp. 337-346.

[13] M. Loper, N. Mahmood, J. Romero, G. Pons-Moll, and M. J. Black, "Smpl: A skinned multi-person linear model," ACM TOG, vol. 34, no. 6, pp. 1-16, 2015.

[14] S. Zuffi, A. Kanazawa, T. Berger-Wolf, and M. J. Black, "Three-d safari: Learning to estimate zebra pose, shape, and texture from images," in IEEE CVPR, 2019, pp. 5359-5368.

[15] G. Pavlakos, V. Choutas, N. Ghorbani, T. Bolkart, A. A. Osman, D. Tzionas, and M. J. Black, "Expressive body capture: $3 \mathrm{~d}$ hands, face, and body from a single image," in IEEE CVPR, 2019, pp. 10 975-10985. 

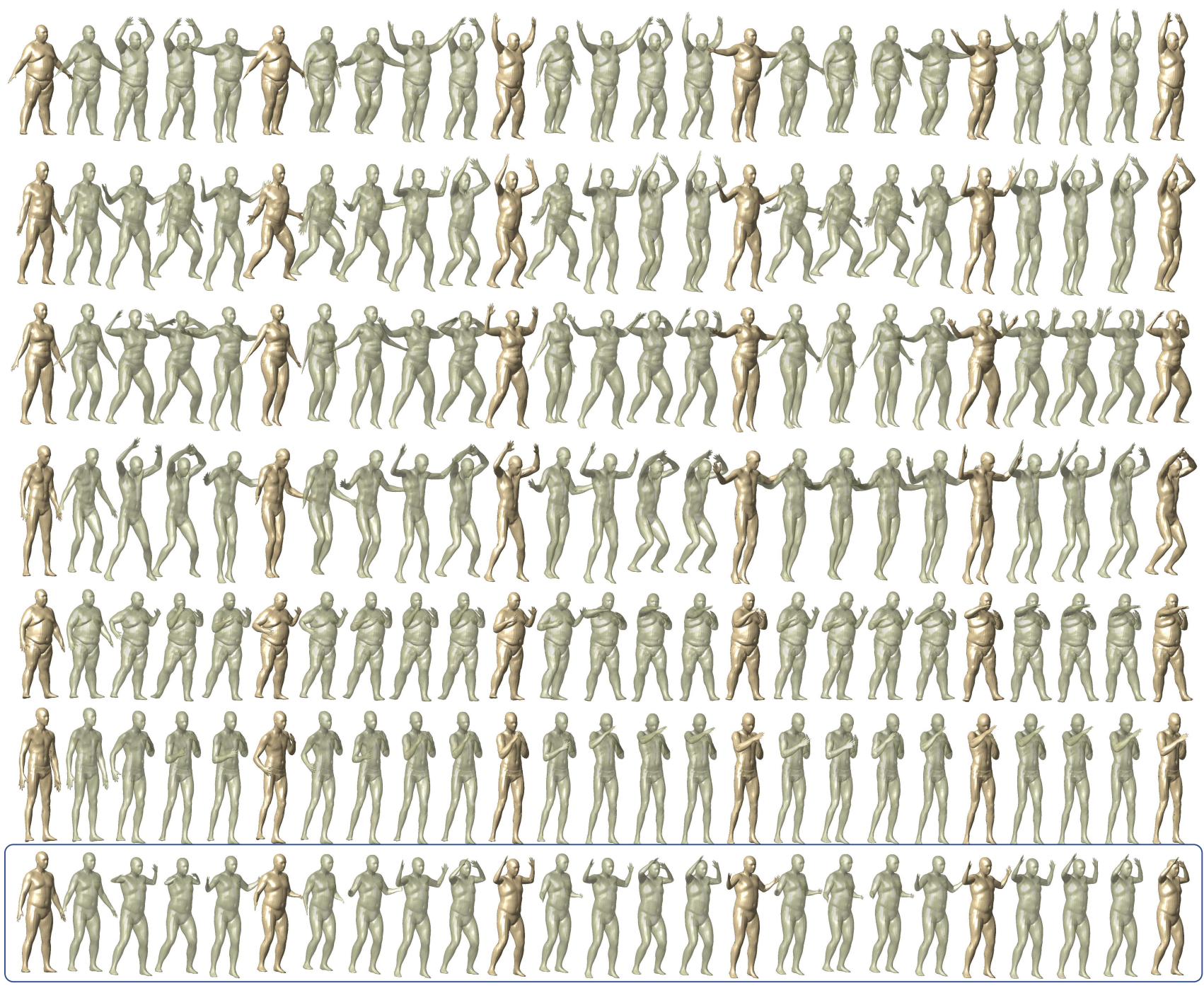

Fig. 12. Co-registration of multiple 4D surfaces. In this example, we consider four human body shapes performing a jumping action (first four rows) and two others performing a punching action (rows 5 and 6). Here, we show the spatiotemporally co-registered 4D surfaces and the 4D mean computed using the proposed algorithm. The supplementary material includes the input 4D surfaces before their spatiotemporal registration. It also includes the full video sequences. The surfaces are from the FAUST dataset.

[16] N. Hesse, S. Pujades, J. Romero, M. J. Black, C. Bodensteiner, M. Arens, U. G. Hofmann, U. Tacke, M. Hadders-Algra, R. Weinberger et al., "Learning an infant body model from rgb-d data for accurate full body motion analysis," in International Conference on Medical Image Computing and Computer-Assisted Intervention. Springer, 2018, pp. 792-800.

[17] S. Kurtek, A. Srivastava, E. Klassen, and H. Laga, "Landmarkguided elastic shape analysis of spherically-parameterized surfaces," CGF, vol. 32, no. 2pt4, pp. 429-438, 2013.

[18] M. Kilian, N. J. Mitra, and H. Pottmann, "Geometric modeling in shape space," in ACM SIGGRAPH, 2007.

[19] Q. Xie, S. Kurtek, H. Le, and A. Srivastava, "Parallel transport of deformations in shape space of elastic surfaces," in IEEE ICCV, December 2013.

[20] Q. Xie, I. Jermyn, S. Kurtek, and A. Srivastava, "Numerical inversion of srnfs for efficient elastic shape analysis of star-shaped objects," in ECCV, 2014, pp. 485-499.

[21] I. H. Jermyn, S. Kurtek, H. Laga, and A. Srivastava, "Elastic shape analysis of three-dimensional objects," Synthesis Lectures on Computer Vision, vol. 12, no. 1, pp. 1-185, 2017.

[22] H. Laga, "A survey on nonrigid 3D shape analysis," in Academic Press Library in Signal Processing, Volume 6. Elsevier, 2018, pp. 261-304.

[23] H. Laga, Y. Guo, H. Tabia, R. B. Fisher, and M. Bennamoun, 3D Shape analysis: fundamentals, theory, and applications. John Wiley \&
Sons, 2018.

[24] A. M. Bronstein, M. M. Bronstein, and R. Kimmel, "Generalized multidimensional scaling: a framework for isometry-invariant partial surface matching," Proceedings of the National Academy of Sciences, vol. 103, no. 5, pp. 1168-1172, 2006.

[25] R. Litman and A. M. Bronstein, "Learning spectral descriptors for deformable shape correspondence," IEEE PAMI, vol. 36, no. 1, pp. 171-180, 2013.

[26] M. Ovsjanikov, M. Ben-Chen, J. Solomon, A. Butscher, and L. Guibas, "Functional maps: a flexible representation of maps between shapes," ACM TOG, vol. 31, no. 4, pp. 1-11, 2012.

[27] M. Ovsjanikov, E. Corman, M. Bronstein, E. Rodolà, M. BenChen, L. Guibas, F. Chazal, and A. Bronstein, "Computing and processing correspondences with functional maps," in SIGGRAPH ASIA 2016 Courses, 2016, pp. 1-60.

[28] R. Anirudh, P. Turaga, J. Su, and A. Srivastava, "Elastic functional coding of human actions: From vector-fields to latent variables," in IEEE CVPR, 2015, pp. 3147-3155.

[29] B. B. Amor, J. Su, and A. Srivastava, "Action recognition using rate-invariant analysis of skeletal shape trajectories," IEEE PAMI, vol. 38, no. 1, pp. 1-13, 2015.

[30] I. Dryden and K. Mardia, Statistical Shape Analysis. John Wiley \& Son, 1998.

[31] A. Srivastava, E. Klassen, S. Joshi, and I. Jermyn, "Shape analysis of elastic curves in euclidean spaces," IEEE PAMI, no. 99, pp. 1-1, 
2011.

[32] V. Debavelaere, S. Durrleman, S. Allassonnière, and A. D. N. Initiative, "Learning the Clustering of Longitudinal Shape Data Sets into a Mixture of Independent or Branching Trajectories," IJCV, pp. 1-16, 2020

[33] A. Bône, O. Colliot, and S. Durrleman, "Learning the Spatiotemporal Variability in Longitudinal Shape Data Sets," IJCV, vol. 128, no. 12, pp. 2873-2896, 2020.

[34] M. F. Beg, M. I. Miller, A. Trouvé, and L. Younes, "Computing large deformation metric mappings via geodesic flows of diffeomorphisms," International journal of computer vision, vol. 61, no. 2, pp. 139-157, 2005.

[35] S. Kurtek, E. Klassen, Z. Ding, S. Jacobson, J. Jacobson, M. Avison, and A. Srivastava, "Parameterization-invariant shape comparisons of anatomical surfaces," Medical Imaging, IEEE Transactions on, vol. 30, no. 3, pp. 849-858, 2011.

[36] J. Su, S. Kurtek, E. Klassen, A. Srivastava et al., "Statistical analysis of trajectories on riemannian manifolds: bird migration, hurricane tracking and video surveillance," The Annals of Applied Statistics, vol. 8, no. 1, pp. 530-552, 2014.

[37] D. T. Robinson, Functional data analysis and partial shape matching in the square root velocity framework. The Florida State University, 2012.

[38] E. Praun and H. Hoppe, "Spherical parametrization and remeshing," vol. 22, 2003, pp. 340-349.

[39] G. Wang, H. Laga, N. Xie, J. Jia, and H. Tabia, "The shape space of 3d botanical tree models," ACM TOG, vol. 37, no. 1, pp. 1-18, 2018.

[40] G. Wang, H. Laga, J. Jia, S. J. Miklavcic, and A. Srivastava, "Statistical analysis and modeling of the geometry and topology of plant roots," J. of Theoretical Biology, vol. 486, p. 110108, 2020.

[41] X. Han, H. Laga, and M. Bennamoun, "Image-based 3d object reconstruction: State-of-the-art and trends in the deep learning era," IEEE PAMI, 2019.

[42] H. Laga, L. V. Jospin, F. Boussaid, and M. Bennamoun, "A survey on deep learning techniques for stereo-based depth estimation," IEEE PAMI, 2020. 


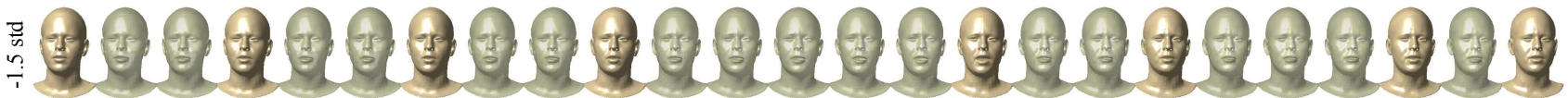

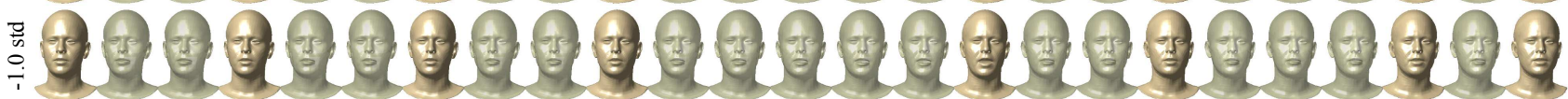

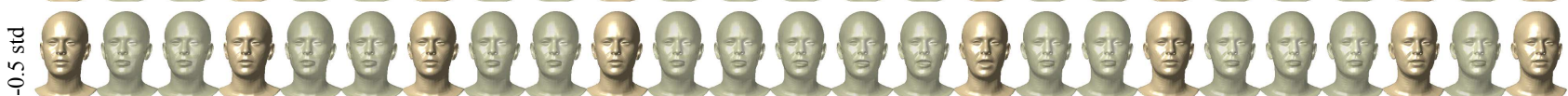
:

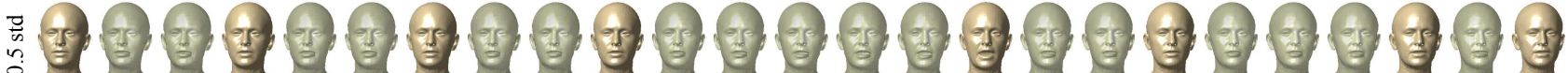
-

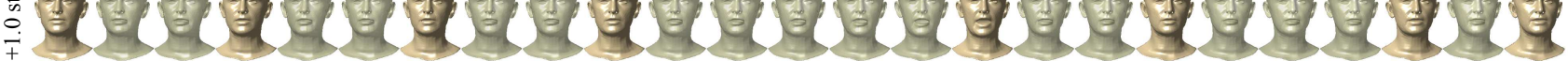

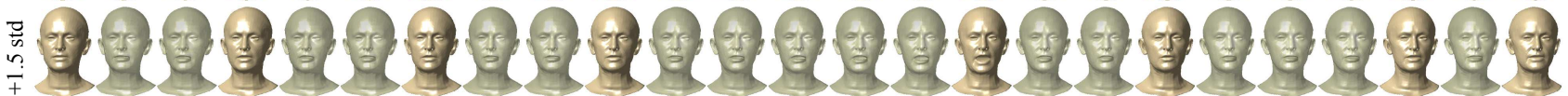

(a) First mode of variation.

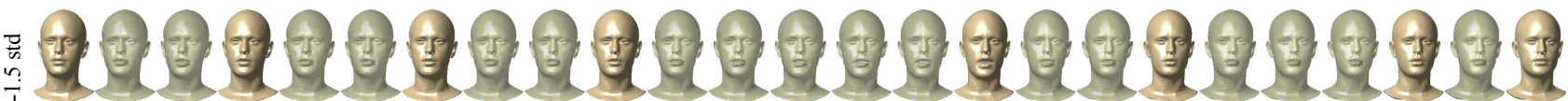

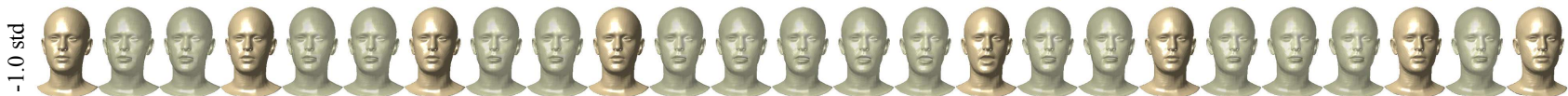

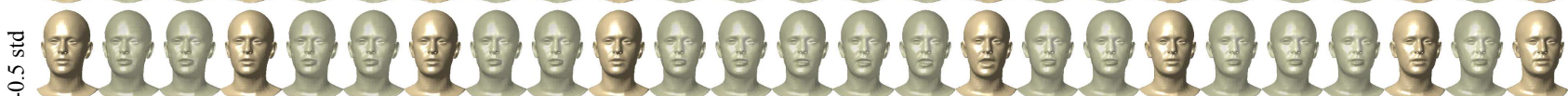

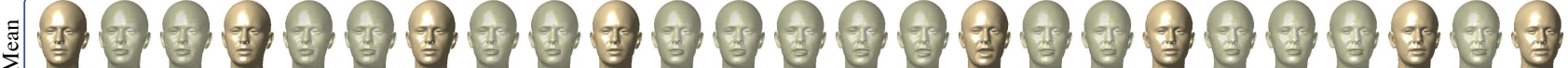
1 309

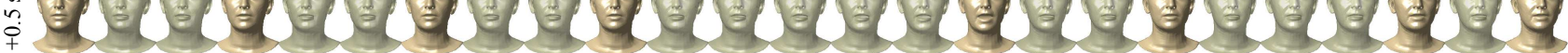

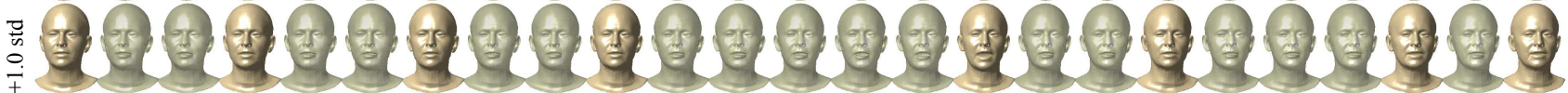

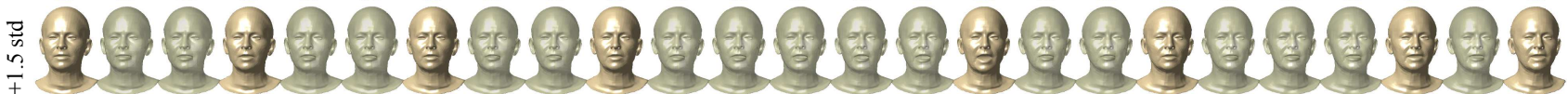

(b) Second mode of variation.

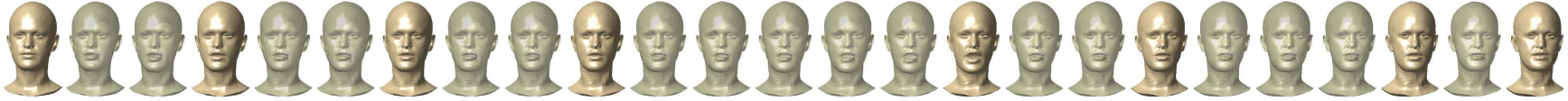

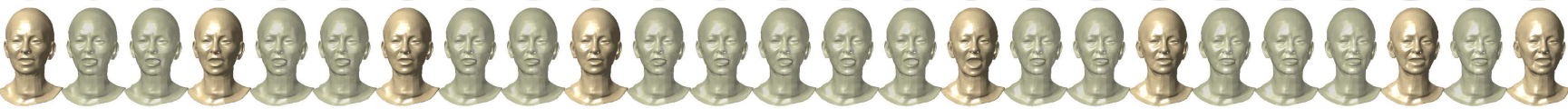

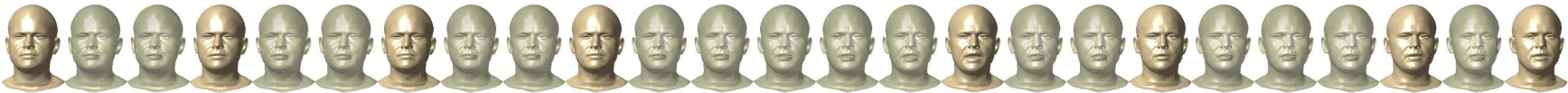
-

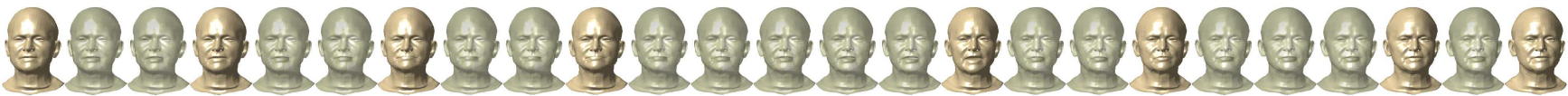

(c) Five randomly synthesized $4 \mathrm{D}$ faces.

Fig. 13. Two principal modes of variation (the mean 4D surface is highlighted in the middle). Each row corresponds to one 4D surface sampled between -1.5 to 1.5 standard deviations along the principal mode of variation. We refer the reader to the supplementary material, which shows the input 4D faces (before their spatiotemporal registration). It also includes more modes of variation and random samples, as well as the full video sequences. 